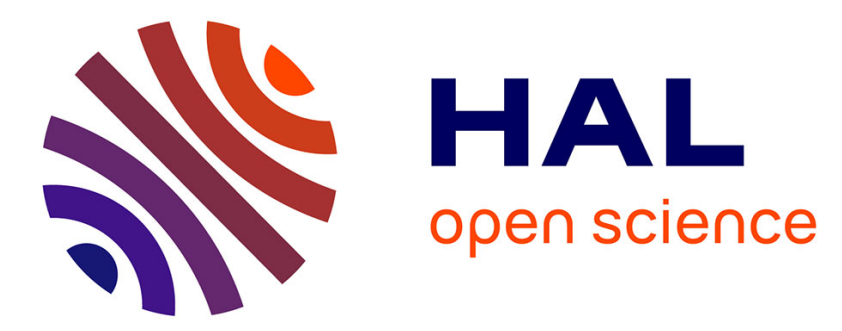

\title{
Dynamique des gaz quantiques (particules discernables sans spin)
}

Pierre-Jean Nacher, Geneviève Tastevin, Franck Laloë

\section{To cite this version:}

Pierre-Jean Nacher, Geneviève Tastevin, Franck Laloë. Dynamique des gaz quantiques (particules discernables sans spin). Journal de Physique, 1989, 50, pp.1907. hal-00001436

\section{HAL Id: hal-00001436 \\ https://hal.science/hal-00001436}

Submitted on 9 Apr 2004

HAL is a multi-disciplinary open access archive for the deposit and dissemination of scientific research documents, whether they are published or not. The documents may come from teaching and research institutions in France or abroad, or from public or private research centers.
L'archive ouverte pluridisciplinaire HAL, est destinée au dépôt et à la diffusion de documents scientifiques de niveau recherche, publiés ou non, émanant des établissements d'enseignement et de recherche français ou étrangers, des laboratoires publics ou privés. 


\title{
DYNAMIQUE DES GAZ QUANTIQUES (PARTICULES DISCERNABLES SANS SPIN)
}

\author{
P.J. Nacher, G. Tastevin et F.Laloë (*)
}

Laboratoire de Physique de l'ENS, 24 rue Lhomond, F 75005 Paris, France

Résumé : Nous appliquons les résultats de deux articles précédents, en particulier une équation cinétique généralisant l'équation de Boltzmann, à l'étude des gaz dilués quantiques hors d'équilibre. Par une simple extension de la méthode de Chapman Enskog appliquée à cette équation cinétique, nous calculons les propriétés hydrodynamiques du gaz. A l'ordre le plus bas (hydrodynamique d'Euler), nous obtenons des résultats pour la pression, la vitesse du son et la capacité calorifique qui, contrairement à ceux de la théorie de Boltzmann, contiennent les secondes corrections du viriel. A l'ordre suivant (hydrodynamique visqueuse, ou de Navier Stokes), nous obtenons les corrections du premier ordre en densité aux coefficients de transport, et montrons qu'il peut exister un terme de seconde viscosité, quadratique en densité.

Ce formalisme donne accès aux parties des corrections du viriel des coefficients de transport qui dépendent des collisions à deux corps, mais pas à celles qui sont dues aux collisions ternaires. Il est quantique et permet de réobtenir l'expression de Beth et Uhlenbeck de la seconde correction du viriel en fonction des déphasages.

Abstract: We apply the results obtained in two preceding articles, in particular a kinetic equation which generalizes the Boltzmann equation, to the study of dilute quantum gases out of equilibrium. Extending the Chapman Enskog method to this equation, and taking into account the specific expressions of the locally conserved quantities in this theory, we calculate the hydrodynamical properties of the gas. To lowest order (Euler hydrodynamics), we obtain results which include second virial corrections to the pressure, sound velocity and specific heat, which are not accessible within the usual frame of the the Boltzmann equation. To first order (viscous, or Navier Stokes, hydrodynamics), we get linear density corrections to transport coefficients. We also show that bulk viscosity may exist, being proportional to the square of density.

This formalism gives access to the part of the second virial corrections to transport coefficients which correspond to transport by binary collisions, but ignores the modifications of the damping of currents due to three body interactions (although it does provide virial corrections to the damping of non hydrodynamical variables). It is purely quantum and, at equilibrium, allows one to recover the Beth Uhlenbeck expression of the second virial correction to the pressure in terms of the collision phase shifts. It will be extended to spin and statistics in a forthcoming article.

(*) Membres du Laboratoire de Spectroscopie Hertzienne de l'ENS, associé au CNRS n 18.

Le Journal de Physique, 50, page 1907 (1989) 


\section{INTRODUCTION}

Dans deux articles A et B précédents [1],[2], nous avons posé les bases d'une étude de la cinétique des gaz dilués qui va au delà de ce que permet l'équation de Boltzmann, car elle tient compte de façon (quasi) exacte de l'ensemble des effets des collisions à deux corps, y compris des fortes corrélations introduites à courte distance par le potentiel d'interaction. La première étape, dans l'article A, a été la définition d'une transformée de Wigner modifiée, la transformée "libre"; cette dernière est mieux adaptée que la transformée habituelle à une hypothèse de factorisation (chaos moléculaire) et donc à l'écriture d'une équation cinétique, tout en conservant un traitement correct des effets de corrélation entre particules à courte distance. La seconde étape (article B) a ensuite été d'écrire explicitement cette équation cinétique et de vérifier qu'elle présente les propriétés requises de conservation des grandeurs hydrodynamiques locales, telles que densité de particules, de courant ou d'énergie.

Le présent article complète en quelque sorte ce programme en calculant explicitement les propriétés hydrodynamiques d'un gaz dans ce formalisme, et en vérifiant qu'elles sont bien compatibles avec ce que l'on sait d'autre part grâce à des calculs valables à l'équilibre : existence d'une seconde correction du viriel dans la pression par exemple.

Après avoir écrit une équation cinétique analogue à celle de l'article $\mathrm{B}$, mais plus commode à utiliser dans la suite, nous commencerons par étudier les propriétés d'un gaz à l'équilibre et montrerons comment le formalisme que nous avons développé permet de retrouver ces corrections dans un cadre différent. Mais l'intérêt de ce formalisme est qu'il donne accès aux mêmes types de correction hors d'équilibre; il permet ainsi de calculer "ab initio", dans un cadre entièrement quantique, certaines corrections du viriel aux coefficients de transport. Mathématiquement, ces corrections apparaissent lorsqu'on généralise à cette équation cinétique la méthode de Chapman et Enskog [3] [4]; elles sont des conséquences à la fois d'un terme source linéarisé qui est plus riche que celui de la théorie standard, et d'expressions différentes des courants en fonction de la distribution $f$ qui apparaît dans l'équation cinétique. Physiquement, elles correspondent au transport "par les collisions" qui se produit lorsque le potentiel a une portée non nulle; un tel effet, classiquement, tend à augmenter les flux d'impulsion et d'énergie [5], mais notre formalisme en permet une étude quantique détaillée. En revanche, puisque notre approche laisse totalement de côté les collisions à trois corps, dont l'effet tend d'ailleurs classiquement à s'opposer au transport par les collisions [5], elle est essentiellement incapable de fournir une expression complète de l'ensemble des secondes corrections du viriel aux coefficients de transport à toute température.

Rappelons un certain nombre d'autres limitations fondamentales de cette approche: les états liés ne sont pas pris en compte, ce qui en fait ne serait pas fondamentalement impossible mais conduirait à introduire plusieurs équations cinétiques couplées (autant que de niveaux liés); les collisions successives sont traitées comme indépendantes (milieu dilué; les "effets de cage" ne sont pas traités); le rôle des spins et de l'indiscernabilité des particules est laissé de côté: il fera l'objet de l'article D suivant.

Nous commençons, au $\S 1$, par réécrire sous une forme plus commode l'équation cinétique et les diverses grandeurs hydrodynamiques définies dans l'article B; nous étudierons au $\S 2$ le cas simple où le système est uniforme dans l'espace, et où la fonction de distribution tend rapidement vers une gaussienne; l'étude détaillée des propriétés à l'équilibre du système sera le point de départ de la suite des raisonnements. Dans le $\S 3$ nous aborderons la systématique du développement de Chapman Enskog, qui part d'une définition adaptée à notre cas des variables lentes et rapides. Le $\S 4$ est consacré à l'étude à l'ordre le plus bas (hydrodynamique d'Euler), tandis que dans le $\S 5$ nous calculerons le terme du premier ordre et les corrections visqueuses et calorifiques qu'il introduit (hydrodynamique de Navier-Stokes). Notons au passage que, certains des calculs de cet article étant techniquement assez lourds, nous les avons effectués grâce à un programme d'Algèbre 
Assistée par Ordinateur. Par exemple, les résultats (132) et (139) ont été obtenus de cette façon.

\section{REECRITURE DE L'EQUATION CINETIQUE}

Rappelons la forme de l'équation dynamique établie dans l'article B:

$$
\left[\frac{\partial}{\partial t}+\frac{\mathbf{p}}{m} \cdot \nabla\right] f(\mathbf{p})=I_{B}[f(\mathbf{p})]+K_{\text {coll }}[f(\mathbf{p})]+\delta I_{B}[f(\mathbf{p})]+\delta K_{\text {coll }}[f(\mathbf{p})] \quad(1-a)
$$

avec:

$$
\begin{aligned}
K_{\text {coll } l}[f(\mathbf{p})] & =G_{\text {coll }}^{\xi}[f(\mathbf{p})]+G_{\text {coll }}^{\Delta}[f(\mathbf{p})] \\
\delta I_{B}[f(\mathbf{p})] & =-\int d^{3} q \Xi\left(\frac{q}{2 \hbar}\right)\left\{f(\mathbf{p}-\mathbf{q})\left(I_{B}+\delta I_{B}\right)[f(\mathbf{p})]+f(\mathbf{p})\left(I_{B}+\delta I_{B}\right)[f(\mathbf{p}-\mathbf{q})]\right\} \\
\delta K_{\text {coll }}[f(\mathbf{p})] & =-\int d^{3} q \Xi\left(\frac{q}{2 \hbar}\right)\left\{f(\mathbf{p}-\mathbf{q})\left(K_{\text {coll }}+\delta K_{\text {coll }}\right)[f(\mathbf{p})]+f(\mathbf{p})\left(K_{\text {coll }}+\delta K_{\text {coll }}\right)[f(\mathbf{p}-\mathbf{q})]\right\}
\end{aligned}
$$

Les notations sont les mêmes que dans l'article $\mathrm{B}$ et nous ne les expliciterons pas à ce stade. Mentionnons simplement que $I_{B}$ désigne l'opérateur de collision de Boltzmann habituel, et que l'opérateur $K_{\text {coll }}$, dont l'expression sera rappelée plus bas (formules (100) et (101)), fait intervenir quatre fonctions du potentiel atomique:

$$
\xi_{F}(k), \Delta_{F}(k), \xi_{k}(k, \theta), \Delta_{k}(k, \theta)
$$

Les deux premières ne dépendent que d'une impulsion, les dernières de deux impulsions relatives. On confondra pour simplifier dépendance en nombre d'onde $k$ ou en impulsion $q=2 \hbar k$. Rappelons en outre la définition du coefficient $\Xi$ (article B, formules (14-a) et (37)):

$$
\Xi(k)=-2 \xi_{F}(k)-2 \frac{\Delta_{F}(k)}{\hbar k}+2 \int d^{2} \hat{k}^{\prime} \xi_{k}(k, \theta)=\frac{2 \pi}{k^{2}} \sum_{l}(2 l+1) \delta_{l}^{\prime}(k) \quad(1-d)
$$

qui joue un rôle important dans toute la suite.

Il ressort de l'analyse de l'article B qu'il peut être plus commode d'étudier l'évolution dynamique de la fonction de distribution totale $g(\mathbf{r}, \mathbf{p}, t)$ définie par:

$$
g(\mathbf{r}, \mathbf{p}, t)=f(\mathbf{r}, \mathbf{p}, t)+\delta f(\mathbf{r}, \mathbf{p}, t)
$$

avec, en n'explicitant plus les dépendances en $\mathbf{r}$ et $t$ :

$$
\delta f(\mathbf{p})=f(\mathbf{p}) \int d^{3} q \Xi(q) f(\mathbf{p}-\mathbf{q})
$$

Notant $D_{\mathbf{p}}$ l'opérateur:

$$
D_{\mathbf{p}}=\frac{\partial}{\partial t}+\frac{\mathbf{p}}{m} \cdot \nabla
$$

l'évolution de $\delta f$ peut être décomposée de la manière suivante:

$$
\begin{aligned}
D_{\mathbf{p}}[\delta f(\mathbf{p})] & =D_{\mathbf{p}}[f(\mathbf{p})] \int d^{3} q \Xi(q) f(\mathbf{p}-\mathbf{q})+f(\mathbf{p}) \int d^{3} q \Xi(q) D_{\mathbf{p}-\mathbf{q}}[f(\mathbf{p}-\mathbf{q})] \\
& +f(\mathbf{p}) \int d^{3} q \Xi(q) \frac{\mathbf{q}}{m} \cdot \nabla f(\mathbf{p}-\mathbf{q})
\end{aligned}
$$


En utilisant (1-a) et (1-b), on peut vérifier que:

$$
D_{\mathbf{p}}[\delta f(\mathbf{p})]=-\delta I_{B}[f(\mathbf{p})]-\delta K_{\text {coll }}[f(\mathbf{p})]+f(\mathbf{p}) \int d^{3} q \Xi(q) \frac{\mathbf{q}}{m} \cdot \nabla f(\mathbf{p}-\mathbf{q})
$$

L'évolution de $g$ s'écrit alors simplement:

$$
D_{\mathbf{p}}[g(\mathbf{p})]=I_{B}[f(\mathbf{p})]+K_{\text {coll }}[f(\mathbf{p})]+R_{\Xi}[f(\mathbf{p})]
$$

où $R_{\Xi}$ est défini par:

$$
R_{\Xi}[f(\mathbf{p})]=f(\mathbf{p}) \int d^{3} q \Xi(q) \frac{\mathbf{q}}{m} \cdot \nabla f(\mathbf{p}-\mathbf{q})
$$

L'équation cinétique (6) a l'avantage d'être formellement plus simple que (1-a): n'y figurent plus les termes correctifs $\delta I_{B}$ et $\delta K_{\text {coll }}$. Toutefois ce n'est pas une équation fermée pour $g$, puisque $f$ figure au second membre. Les équations (6) et (7) sont le point de départ de tous les calculs qui suivent.

Les densités de particules $N(\mathbf{r}, t)$, de courant $\mathbf{J}(\mathbf{r}, t)$ et d'énergie $U(\mathbf{r}, t)$ définies dans l'article $\mathrm{B}$ s'expriment de manière simple en fonction de $g$ :

$$
\begin{aligned}
N(\mathbf{r}, t) & =\int d^{3} p g(\mathbf{r}, \mathbf{p}, t) \\
\mathbf{J}(\mathbf{r}, t) & =\int d^{3} p \frac{\mathbf{p}}{m} g(\mathbf{r}, \mathbf{p}, t) \\
U(\mathbf{r}, t) & =\int d^{3} p \frac{p^{2}}{2 m} g(\mathbf{r}, \mathbf{p}, t)
\end{aligned}
$$

Les lois de conservation correspondantes nous ont conduits à définir des formes particulières pour le tenseur des contraintes $\overline{\mathbf{Q}}$ et le courant de chaleur $\mathbf{J}_{U}$ ( expressions (54) et (68) dans l'article B ). En y substituant $g-\delta f$ à $f$, ces relations peuvent être réécrites sous la forme:

$$
\overline{\mathbf{Q}}=\int d^{3} p \frac{\mathbf{p} \otimes \mathbf{p}}{m} g(\mathbf{p})+\overline{\mathbf{Q}}^{(F)}+\overline{\mathbf{Q}}^{(k)}+\overline{\mathbf{Q}}^{(\Xi)}
$$

où $\overline{\mathbf{Q}}^{(F)}$ et $\overline{\mathbf{Q}}^{(k)}$ sont les tenseurs notés $\delta \overline{\mathbf{Q}}\left(\Delta_{F}\right)$ et $\delta \overline{\mathbf{Q}}\left(\Delta_{k}\right)$ dans l'article $\mathrm{B}$, donnés par:

$$
\begin{aligned}
& \overline{\mathbf{Q}}^{(F)}=-\frac{1}{2 m} \int d^{3} p \int d^{3} q \frac{1}{q} \Delta_{F}(q)\left(3 \mathbf{q} \otimes \mathbf{q}-q^{2} \overline{\mathbf{1}}\right) f(\mathbf{p}) f(\mathbf{p}-\mathbf{q}) \\
& \overline{\mathbf{Q}}^{(k)}=-\frac{1}{2 m} \int d^{3} p \int d^{3} q \int d^{2} \hat{q}^{\prime} \Delta_{k}(q, \theta) \mathbf{q} \otimes\left[\mathbf{q}^{\prime}-\left(\hat{q} \cdot \hat{q}^{\prime}\right) \mathbf{q}\right] f\left(\mathbf{1}^{\prime}\right) f\left(\mathbf{2}^{\prime}\right)
\end{aligned}
$$

où on note $\mathbf{1}^{\prime}$ et $\mathbf{2}^{\prime}$ les impulsions $\mathbf{p}-\mathbf{q} / 2+\mathbf{q}^{\prime} / 2$ et $\mathbf{p}-\mathbf{q} / 2-\mathbf{q}^{\prime} / 2$ et où on pose:

$$
\overline{\mathbf{Q}}^{(\Xi)}=-\frac{1}{4 m} \int d^{3} p \int d^{3} q \Xi(q) \mathbf{q} \otimes \mathbf{q} f(\mathbf{p}) f(\mathbf{p}-\mathbf{q})
$$

Le courant de chaleur s'écrit:

$$
\mathbf{J}_{U}=\int d^{3} p \frac{p^{2}}{2 m^{2}} \mathbf{p} g(\mathbf{p})+\mathbf{J}_{U}^{(F)}+\mathbf{J}_{U}^{(k)}+\mathbf{J}_{U}^{(\Xi)}
$$


où $\mathbf{J}_{U}^{(F)}$ et $\mathbf{J}_{U}^{(k)}$, notés $\delta \mathbf{J}_{U}\left(\Delta_{F}\right)$ et $\delta \mathbf{J}_{U}\left(\Delta_{k}\right)$ dans l'article $\mathrm{B}$, sont donnés par:

$$
\begin{aligned}
\mathbf{J}_{U}^{(F)} & =-\frac{1}{2 m^{2}} \int d^{3} p \int d^{3} q \frac{1}{q} \Delta_{F}(q)\left[\left(3\left(\mathbf{p}-\frac{\mathbf{q}}{2}\right) \cdot \mathbf{q}\right) \mathbf{q}-q^{2}\left(\mathbf{p}-\frac{\mathbf{q}}{2}\right)\right] f(\mathbf{p}) f(\mathbf{p}-\mathbf{q}) \\
\mathbf{J}_{U}^{(k)} & =-\frac{1}{2 m^{2}} \int d^{3} p \int d^{3} q \int d^{2} \hat{q}^{\prime} \Delta_{k}(\theta)\left(\mathbf{p}-\frac{\mathbf{q}}{2}\right) \cdot \mathbf{q}\left[\mathbf{q}^{\prime}-\left(\hat{q} \cdot \hat{q}^{\prime}\right) \mathbf{q}\right] f\left(\mathbf{1}^{\prime}\right) f\left(\mathbf{2}^{\prime}\right)
\end{aligned}
$$

et où on a posé:

$$
\mathbf{J}_{U}^{(\Xi)}=-\frac{1}{4 m^{2}} \int d^{3} p f(\mathbf{p}) \int d^{3} q \Xi(q)\left(\mathbf{p}-\frac{\mathbf{q}}{2}\right) \cdot \mathbf{q} \mathbf{q} f(\mathbf{p}) f(\mathbf{p}-\mathbf{q})
$$

Tout comme l'équation cinétique (6), ces expressions font intervenir à la fois $g$ et $f$. Elles ont été déduites des résultats de l'article $\mathrm{B}$, mais peuvent également être directement établies à partir de l'équation (6) pour assurer la conservation locale des trois quantités $N, \mathbf{J}$ et $U$. Le détail de ces calculs est présenté dans l'appendice C1.

\section{SYSTEME UNIFORME DANS L'ESPACE \\ 2.1 EVOLUTION TEMPORELLE}

Pour un système uniforme dans l'espace, les termes en $K_{\text {coll }}$ et $\delta K_{\text {coll }}$ dans l'équation cinétique (1-a) sont nuls, et celle-ci se réduit à:

$$
\frac{\partial}{\partial t} f(\mathbf{p})=I_{B}[f(\mathbf{p})]+\delta I_{B}[f(\mathbf{p})]
$$

La seule différence avec l'équation de Boltzmann habituelle est la présence du terme $\delta I_{B}$, qui rend le second membre de degré trois en $f$, et non deux. Formellement l'équation (6) devient, elle, encore plus simple puisqu'elle s'écrit:

$$
\frac{\partial}{\partial t} g(\mathbf{p})=I_{B}[f(\mathbf{p})]
$$

L'équation (19) reste cependant assez semblable à l'équation différentielle habituelle; en particulier, le second membre s'annule également lorsque $f$ est une fonction gaussienne. L'étude mathématique générale de ce type d'équation non linéaire pose déjà des problèmes difficiles pour l'équation de Boltzmann habituelle [6] [7] [8] ; la présence de $\delta I_{B}$ ne simplifie pas la situation -sans toutefois la bouleverser radicalement dans la mesure où ce terme supplémentaire est supposé petit devant $I_{B}$-et nous nous contenterons d'admettre sans démonstration que, quelles que soient les conditions initiales, $f$ tend toujours au bout d'un temps de l'ordre de quelques fois le temps de collision $\tau_{c}$, vers une fonction gaussienne:

$$
f^{e q}(\mathbf{p})=N_{L}\left(\frac{\beta}{2 \pi m}\right)^{3 / 2} e^{-\beta(\mathbf{p}-m \mathbf{V})^{2} / 2 m}
$$

où $\beta=\left(k_{B} T\right)^{-1}$. L'équation (20) montre que la fonction $g$ relaxe, sur la même échelle de temps, vers sa forme d'équilibre qui est:

$$
g^{e q}(\mathbf{p})=f^{e q}(\mathbf{p})+f^{e q}(\mathbf{p}) \int d^{3} q \Xi(q) f^{e q}(\mathbf{p}-\mathbf{q})
$$


Nous pouvons donc à présent calculer toutes les quantités physiques introduites dans l'article B, pour la solution stationnaire (22) dont on connait la forme explicite.

\subsection{CALCUL DES DIFFERENTES QUANTITES PHYSIQUES}

Les différentes quantités à calculer font intervenir les sommes sur $\mathbf{p}$ de la fonction $g$, multipliée par $1, \mathbf{p}, p^{2}, p^{2} \mathbf{p}$, etc...Il est alors plus commode d'introduire une famille de fonctions gaussiennes $\Phi_{a}$, définies par:

$$
\Phi_{a}(\mathbf{x})=N_{L}\left(\frac{\beta}{2 \pi a}\right)^{3 / 2} e^{-\beta \mathbf{x}^{2} / 2 a}
$$

La solution stationnaire s'écrit simplement:

$$
f^{e q}(\mathbf{p})=\Phi_{m}(\mathbf{p}-m \mathbf{V})
$$

et

$$
g^{e q}(\mathbf{p})=\Phi_{m}(\mathbf{p}-m \mathbf{V})+\Phi_{m}(\mathbf{p}-m \mathbf{V}) \int d^{3} q \Xi(q) \Phi_{m}(\mathbf{p}-m \mathbf{V}-\mathbf{q}) \quad(24-b)
$$

ou encore:

$$
g^{e q}(\mathbf{p})=\Phi_{m}(\mathbf{p}-m \mathbf{V})+\int d^{3} q \Xi(q) \Phi_{m / 2}\left(\mathbf{p}-\frac{\mathbf{q}}{2}-m \mathbf{V}\right) \Phi_{2 m}(\mathbf{q})
$$

La formulation (24-c) fait mieux ressortir la possibilité - abondamment utilisée dans les lignes qui suivent - de transformer pour la deuxième partie du membre de droite les sommes sur $\mathbf{p}$ et $\mathbf{q}$ en sommes sur $\mathbf{p}^{\prime \prime}$ et $\mathbf{q}$, où on a posé:

$$
\mathbf{p}^{\prime \prime}=\mathbf{p}-\frac{\mathbf{q}}{2}-m \mathbf{V}
$$

Alors nous pourrons aisément séparer les deux intégrales, de sorte que toutes les quantités à calculer s'exprimeront simplement à partir des différents moments des fonctions $\Phi_{a}$, ou $\Xi \times \Phi_{a}$. Pour alléger la présentation, nous avons écrits et regroupés ceux-ci dans l'appendice C2, auquel nous ferons souvent référence dans ce paragraphe.

Notons dès à présent que tous les calculs présentés ici reposent exclusivement sur le caractère gaussien de $f^{e q}$, et notamment sur son invariance par rotation dans l'espace des impulsions lorsqu'on se place dans le référentiel où la vitesse moyenne des particules est nulle (symétrie sphérique des fonctions $\Phi_{a}$ ). Le caractère uniforme de la solution stationnaire n'intervient pas directement dans le calcul de $N, \mathbf{J}, U, \overline{\mathbf{Q}}$, etc...; tous les résultats que nous allons obtenir pourront être utilisés aussi dans les cas où la fonction $f$ ne serait que localement gaussienne.

\subsubsection{DENSITE DE PARTICULES}

L'équation (8) s'écrit ici:

$$
N=\int d^{3} p \Phi_{m}(\mathbf{p}-m \mathbf{V})+\int d^{3} p \int d^{3} q \Xi(q) \Phi_{m / 2}\left(\mathbf{p}-\frac{\mathbf{q}}{2}-m \mathbf{V}\right) \Phi_{2 m}(\mathbf{q})
$$

En passant de la variable $\mathbf{p}$ à la variable $\mathbf{p}^{\prime}$, avec:

$$
\mathbf{p}^{\prime}=\mathbf{p}-m \mathbf{V}
$$

pour le premier terme, et à la variable $\mathbf{p}^{\prime \prime}$ - formule (25) - pour le second, on obtient immédiatement:

$$
N=N_{L}+N_{L}^{2} \Sigma_{0}
$$


où $\Sigma_{0}$ est l'intégrale définie par la formule (C2-6) dans l'appendice $\mathrm{C} 2$. Elle ne dépend que de la température, de la masse $m$ et du potentiel d'interaction.

\subsubsection{DENSITE DE COURANT}

On peut réécrire (9) sous la forme:

$$
\mathbf{J}=\int d^{3} p \frac{\mathbf{p}-m \mathbf{V}}{m} g(\mathbf{p})+\mathbf{V} \int d^{3} p g(\mathbf{p})
$$

En utilisant l'expression (24-b) pour $g^{e q}$, on voit facilement que le premier terme est nul, puisqu'il s'écrit:

$$
\int d^{3} p^{\prime} \frac{\mathbf{p}^{\prime}}{m} \Phi_{m}\left(\mathbf{p}^{\prime}\right)+\int d^{3} p^{\prime} \int d^{3} q \Xi(q) \frac{\mathbf{p}^{\prime}}{m} \Phi_{m}\left(\mathbf{p}^{\prime}\right) \Phi_{m}\left(\mathbf{p}^{\prime}-\mathbf{q}\right)
$$

et que les fonctions $\Phi_{m}$ sont paires (effectuer le changement de $\mathbf{p}^{\prime}$ en $-\mathbf{p}^{\prime}$ pour la première intégrale, de $\mathbf{p}^{\prime}$ en $-\mathbf{p}^{\prime}$ et de $\mathbf{q}$ en $-\mathbf{q}$ pour la seconde).

Le second terme contribue seul et donne:

$$
\mathbf{J}=N \mathbf{V}
$$

\subsubsection{DENSITE D'ENERGIE}

De la même manière, nous transformons (10) en:

$$
U=\int d^{3} p \frac{(\mathbf{p}-m \mathbf{V})^{2}}{2 m} g(\mathbf{p})+\int d^{3} p \mathbf{p} \cdot \mathbf{V} g(\mathbf{p})-\int d^{3} p \frac{1}{2} m V^{2} g(\mathbf{p})
$$

Pour $g=g^{e q}$, la deuxième intégrale contribue pour:

$$
m \mathbf{J} \cdot \mathbf{V}=m N V^{2}
$$

et la troisième pour:

$$
-\frac{1}{2} m N V^{2}
$$

Il ne reste qu'à calculer la première intégrale, notée $W$ :

$$
W=\int d^{3} p \frac{(\mathbf{p}-m \mathbf{V})^{2}}{2 m} g^{e q}(\mathbf{p})
$$

Elle s'écrit:

$$
\begin{aligned}
W= & \int d^{3} p^{\prime} \frac{p^{\prime 2}}{2 m} \Phi_{m}\left(\mathbf{p}^{\prime}\right) \\
+\int d^{3} p & \int d^{3} q\left[\frac{(\mathbf{p}-m \mathbf{V}-\mathbf{q} / 2)^{2}}{2 m}+\frac{(\mathbf{p}-m \mathbf{V}-\mathbf{q} / 2) \cdot \mathbf{q}}{2 m}+\frac{q^{2}}{8 m}\right] \\
& \times \Xi(q) \Phi_{m / 2}\left(\mathbf{p}-\frac{\mathbf{q}}{2}-m \mathbf{V}\right) \Phi_{2 m}(\mathbf{q})
\end{aligned}
$$

La contribution du premier terme dans (35) donne - voir appendice C2 -:

$$
\frac{3}{2 \beta} N_{L}
$$


ce qui n'est pas sans évoquer un résultat classique en théorie de Boltzmann.

Pour le second terme, nous avons explicitement fait apparaître trois morceaux. Le premier vaut:

$$
\left(\int d^{3} p^{\prime \prime} \frac{p^{\prime \prime 2}}{2 m} \Phi_{m / 2}\left(\mathbf{p}^{\prime \prime}\right)\right) \times\left(\int d^{3} q \Xi(q) \Phi_{2 m}(\mathbf{q})\right)=\frac{3}{4 \beta} N_{L}^{2} \Sigma_{0}
$$

le second:

$$
\left(\int d^{3} p^{\prime \prime} \frac{\mathbf{p}^{\prime \prime}}{2 m} \Phi_{m / 2}\left(\mathbf{p}^{\prime \prime}\right)\right) \cdot\left(\int d^{3} q \Xi(q) \mathbf{q} \Phi_{2 m}(\mathbf{q})\right)=0
$$

(nul par parité); et le troisième est égal à:

$$
N_{L} \frac{1}{8 m} \int d^{3} q \Xi(q) q^{2} \Phi_{2 m}(\mathbf{q})
$$

qui, avec la définition (C2-6) de l'appendice C2, s'écrit:

$$
\frac{1}{2 \beta} N_{L}^{2} \Sigma_{1}
$$

Par regroupement, nous obtenons:

$$
W=\frac{3}{2 \beta} N_{L}+\frac{3}{4 \beta} N_{L}^{2} \Sigma_{0}+\frac{1}{2 \beta} N_{L}^{2} \Sigma_{1}
$$

Finalement, compte tenu du fait que:

$$
U=W+\frac{1}{2} N m V^{2}
$$

nous pouvons écrire:

$$
\begin{aligned}
U & =\frac{3}{2 \beta} N_{L}+\frac{3}{4 \beta} N_{L}^{2} \Sigma_{0}+\frac{1}{2 \beta} N_{L}^{2} \Sigma_{1}+\frac{1}{2} m N V^{2} \\
& =\frac{3}{2 \beta} N+\frac{1}{2} m N V^{2}-\frac{3}{4 \beta} N_{L}^{2} \Sigma_{0}+\frac{1}{2 \beta} N_{L}^{2} \Sigma_{1}
\end{aligned}
$$

La relation entre $U$ et $N$ n'est plus aussi simple que la relation entre $U_{L}$ et $N_{L}$, obtenue dans l'article B. Remarquons que (43) peut également se mettre sous la forme suivante:

$$
U=\frac{3}{2 \beta} N+\frac{1}{2} m N V^{2}-\frac{1}{2} N_{L}^{2} \frac{d}{d \beta} \Sigma_{0}
$$

\subsubsection{TENSEUR DES CONTRAINTES ET PRESSION}

Les calculs requis sont sensiblement plus lourds que les précédents. Aussi, nous avons choisi d'en présenter le détail dans l'appendice C3, et de nous contenter de donner ici leurs résultats finals.

Pour le système uniforme considéré, le tenseur des contraintes s'écrit:

$$
\overline{\mathbf{Q}}=\left(\frac{2}{3} U-\frac{1}{3} m N V^{2}-\frac{1}{3 \beta} N_{L}^{2} \Sigma_{1}\right) \overline{\mathbf{1}}+m N \mathbf{V} \otimes \mathbf{V}
$$


Il est à noter que les termes $\overline{\mathbf{Q}}^{(F)}$ et $\overline{\mathbf{Q}}^{(k)}$ sont nuls, de sorte que le terme $-N_{L}^{2} \Sigma_{1} / 3 \beta$ correspond à la contribution de $\overline{\mathbf{Q}}^{(\Xi)}$ seulement.

Compte tenu de (C1-12) et (43), le tenseur des pressions est scalaire et vaut:

$$
P=\frac{1}{\beta} N_{L}+\frac{1}{2 \beta} N_{L}^{2} \Sigma_{0}
$$

\subsubsection{COURANT DE CHALEUR}

Pour un système uniforme, $\mathbf{J}_{T}$ est nul. Plus précisément, nous montrons dans l'appendice C3 que chaque composante $\mathbf{J}_{U}^{(k)}, \mathbf{J}_{U}^{(F)}, \mathbf{J}_{U}^{(\Xi)}$ compense exactement la contribution correspondante $\overline{\mathbf{Q}}_{U}^{(k)} \cdot \mathbf{V}, \overline{\mathbf{Q}}_{U}^{(F)} \cdot \mathbf{V}, \overline{\mathbf{Q}}_{U}^{(\Xi)} \cdot \mathbf{V}$ introduite par le tenseur des contraintes dans l'expression de $\mathbf{J}_{T}$ :

$$
\begin{aligned}
\mathbf{J}_{T} & =\mathbf{J}_{U}-U \mathbf{V}-\overline{\mathbf{P}} \cdot \mathbf{V} \\
& =\mathbf{J}_{U}-U \mathbf{V}-\overline{\mathbf{Q}} \cdot \mathbf{V}+m N(\mathbf{V} \otimes \mathbf{V}) \cdot \mathbf{V}
\end{aligned}
$$

\subsection{EQUATION D'ETAT ET CHALEUR SPECIFIQUE}

Nous pouvons tout de suite écrire l'équation d'état du système, puisque la densité de particules et la pression viennent d'être calculées. Nous partons de l'équation (46), que nous écrivons:

$$
\frac{P}{N k_{B} T}=1-\frac{\Sigma_{0} N_{L}^{2}}{2 N}=1-\frac{\Sigma_{0}}{2} \frac{N}{\left(1+\Sigma_{0} N_{L}^{2}\right)^{2}}
$$

A l'ordre le plus bas, nous obtenons:

$$
\frac{P}{N k_{B} T} \simeq 1-\frac{\Sigma_{0}}{2} N
$$

Le second coefficient du viriel est donc:

$$
B_{2}(T)=-\frac{1}{2} \Sigma_{0}
$$

Compte tenu des relations (C2-6) ou (C2-8) de l'appendice $\mathrm{C} 2$, et moyennant un calcul classique, on obtient:

$$
\begin{aligned}
\Sigma_{0} & =\left[\pi m k_{B} T\right]^{-3 / 2} \int d^{3} p \Xi\left(\frac{p}{\hbar}\right) e^{-\beta p^{2} / m} \\
& =2^{3 / 2} \pi^{-1}\left[\lambda_{T}\right]^{3} \int_{0}^{\infty} d k e^{-\beta \hbar^{2} k^{2} / m} \sum_{l}(2 l+1) \delta_{l}^{\prime}(k)
\end{aligned}
$$

où la longueur d'onde thermique a la définition habituelle:

$$
\lambda_{T}=h\left(2 \pi m k_{B} T\right)^{-1 / 2}
$$

Nous retrouvons ainsi un résultat connu [9], dû à Beth et Uhlenbeck [10], mais dans un contexte différent qui donne plus d'information sur l'origine physique des effets. Les formules (18-e) et (18-f) de l'article B ainsi que la formule (1-d) permettent de dégager les contributions de la diffusion vers l'avant et de la diffusion latérale, ou encore de séparer celle des termes de retard pur (en $\xi$ ) de celle des termes en $\Delta$ (champ moléculaire).

A partir de la densité totale d'énergie (43), on peut calculer la chaleur spécifique. Elle vaut: 


$$
\begin{aligned}
C_{V}=\left(\frac{\partial U}{\partial T}\right)_{N}=\frac{3}{2} k_{B} N_{L} & \left\{1-\frac{1}{4} N_{L} \Sigma_{0}+\frac{1}{3} N_{L} \Sigma_{2}\right. \\
& \left.+\frac{1}{1+2 N_{L} \Sigma_{0}}\left[\frac{3}{2} N_{L} \Sigma_{0}-N_{L} \Sigma_{1}+\frac{3}{2}\left(N_{L} \Sigma_{0}\right)^{2}-\frac{2}{3}\left(N_{L} \Sigma_{1}\right)^{2}\right]\right\}
\end{aligned}
$$

Au premier ordre, nous avons:

$$
C_{V} \simeq \frac{3}{2} k_{B} N_{L}\left(1+\frac{5}{4} N_{L} \Sigma_{0}-N_{L} \Sigma_{1}+\frac{1}{3} N_{L} \Sigma_{2}\right)
$$

ou encore:

$$
\begin{aligned}
C_{V} & \simeq \frac{3}{2} k_{B} N_{L}\left(1+N_{L} \Sigma_{0}+\frac{1}{3} N_{L} \beta^{2} \frac{d^{2}}{d \beta^{2}} \Sigma_{0}\right) \\
& \simeq \frac{3}{2} k_{B} N+\frac{1}{2} N_{L}^{2} \beta^{2} \frac{d^{2}}{d \beta^{2}} \Sigma_{0}
\end{aligned}
$$

Les formules (43) et (50) ne sont pas indépendantes du point de vue de la thermodynamique. Si en effet la fonction de partition d'un système physique est donnée par:

$$
Z=Z_{0}+\Delta Z
$$

où $Z_{0}$ est la fonction de partition d'un gaz parfait classique, on calcule facilement au premier ordre en $\Delta Z$ que:

$$
P=k_{B} T\left(\frac{\partial}{\partial V}\right)_{T} \log Z=N k_{B} T+\Delta P
$$

où on a posé:

$$
\Delta P=k_{B} T\left(\frac{\partial}{\partial V}\right)_{T} \frac{\Delta Z}{Z_{0}}
$$

(conformément à l'usage, nous notons dans ce paragraphe $V$ le volume du système et non la vitesse qui n'intervient pas ici). D'autre part, on a l'égalité suivante:

$$
E=V U=-\left(\frac{\partial}{\partial \beta}\right)_{V} \log Z
$$

qui conduit à:

$$
U=\frac{3}{2} N k_{B} T+\Delta U
$$

avec:

$$
\Delta U=-\frac{1}{V}\left(\frac{\partial}{\partial \beta}\right)_{V} \frac{\Delta Z}{Z_{0}}
$$

Pour finir, en comparant (56) et (59), on a:

$$
\left(\frac{\partial}{\partial \beta}\right)_{V} \frac{\Delta P}{k_{B} T}=\left(\frac{\partial}{\partial V}\right)_{T}(-V \Delta U)
$$


Il est facile de vérifier sur (44) et (49) que les corrections à la densité d'énergie et à la pression vérifient effectivement cette relation. Nous avons ainsi retrouvé dans un cadre différent des corrections du viriel bien connues; cela constitue un test de la validité de notre approche, et en particulier de la définition que nous avons prise pour la température (en termes de $N_{L}$ et $U_{L}$, et non des vraies densités de particules et d'énergie - cf article B).

\section{METHODE DE CHAPMAN ENSKOG: CADRE GENERAL}

\subsection{DEFINITION DES VARIABLES LENTES ET RAPIDES}

L'adaptation de la méthode de Chapman et Enskog à l'équation cinétique (6) demande un certain nombre de modifications liées à la présence de la fonction $f$, dont le lien avec les variables hydrodynamiques est plus complexe que dans le cas habituel. Aussi allons nous rapidement, dans les lignes qui suivent, revenir sur le principe de cette méthode.

Pour des raisons physiques, on s'attend à ce que les véritables variables lentes (hydrodynamiques) du problème soient les quantités réellement conservées par les collisions, à savoir $N, \mathbf{V}$ (ou $\mathbf{J}$ ), et $W$ (ou $U$ ), qui sont liées aux trois premiers moments de $g$, et non $N_{L}$ ni $W_{L}$ (ou $\left.U_{L}\right)$ liées aux moments de $f$; nous verrons dans le $\S 3.2$ que c'est effectivement le cas. D'après les résultats du paragraphe précédent (équations (28) et (41)), on a pour toute situation où $f$ est localement gaussienne:

$$
\begin{aligned}
N & =N_{L}\left(1+N_{L} \Sigma_{0}\right) \\
W & =\frac{3}{2} N_{L} k_{B} T\left(1+\frac{1}{2} N_{L} \Sigma_{0}+\frac{1}{3} N_{L} \Sigma_{1}\right)
\end{aligned}
$$

Inversement, ces équations peuvent être vues comme un système de deux équations non linéaires en $N_{L}$ et $T$ qui fixent ces variables en fonction des variables hydrodynamiques $N$ et $W$. On note $N_{L}^{(0)}$ et $T^{(0)}$ les solutions du système d'équations (61), elles-mêmes des variables lentes:

$$
\begin{aligned}
N_{L}^{(0)} & =N_{L}^{(0)}(N, W) \\
T^{(0)} & =T^{(0)}(N, W)
\end{aligned}
$$

Comme nous supposons que $N_{L} \Sigma_{0}$ et $N_{L} \Sigma_{1}$ sont petits (gaz dilué), la solution (62) reste au voisinage du couple de valeurs triviales $\left(N, 2 W / 3 N k_{B}\right)$ et nous pouvons considérer qu'elle est unique.

Il est donc possible de décrire tout état hydrodynamique du système, caractérisé par ses variables $N(\mathbf{r}, t), \mathbf{V}(\mathbf{r}, t)$ et $W(\mathbf{r}, t)$, par une fonction de distribution localement gaussienne $f^{(0)}(\mathbf{p} ; \mathbf{r}, t)$ donnée par:

$$
f^{(0)}(\mathbf{p})=N_{L}^{(0)}\left(2 \pi m k_{B} T^{(0)}\right)^{-3 / 2} \exp \left\{-\frac{(\mathbf{p}-m \mathbf{V})^{2}}{2 m k_{B} T^{(0)}}\right\}
$$

C'est cette fonction $f^{(0)}$, et la distribution $g^{(0)}$ associée, qui s'expriment en fonction des seules variables lentes, que nous prendrons comme ordre zéro du développement de Chapman Enskog. Elles décrivent correctement les valeurs locales des variables hydrodynamiques, et la forme localement gaussienne de $f^{(0)}$ est la plus commode pour les calculs ultérieurs (c'est en effet $f$ qui figure au second membre de l'équation cinétique).

En ce qui concerne les variables rapides, ce sont celles qui contiennent le reste de l'information qui permet d'obtenir la fonction $f$ (donc $g$ ); elles sont en nombre infini puisque $f$ dépend de la variable continue $\mathbf{p}$. Notons $g^{r a p}$ la contribution à $g$ des variables rapides:

$$
g^{r a p}(\mathbf{r}, \mathbf{p}, t)=g(\mathbf{r}, \mathbf{p}, t)-g^{(0)}[\mathbf{p} ; N(\mathbf{r}, t), \mathbf{V}(\mathbf{r}, t), W(\mathbf{r}, t)]
$$


Il est clair que les valeurs de $g^{r a p}(\mathbf{p})$ pour les différentes valeurs de $\mathbf{p}$ ne sont pas indépendantes puisque, par construction, les distributions $g^{(0)}$ et $g$ donnent les mêmes valeurs aux variables lentes; on a les contraintes:

$$
\int d^{3} p F(\mathbf{p}) g^{r a p}(\mathbf{p})=0 \quad \text { pour } F(\mathbf{p})=1, \mathbf{p} \text { ou } p^{2}
$$

On peut remarquer à ce stade que, si toutes les grandeurs calculées (à l'ordre zéro) à partir de $f^{(0)}$ (telles $N_{L}^{(0)}, W_{L}^{(0)}, T^{(0)} \ldots$ ) sont des fonctions des variables lentes seules, en revanche les grandeurs complètes correspondantes $\left(N_{L}, W_{L}\right.$, et même la température $\left.T\right)$, sont des mélanges de variables lentes et rapides. Ainsi a-t-on pour $N_{L}$ par exemple:

$$
N_{L}=\int d^{3} p\left(f^{(0)}+f^{r a p}\right)=N_{L}^{(0)}+\int d^{3} p f^{r a p}
$$

\subsection{EQUATIONS D'EVOLUTION}

Les équations hydrodynamiques exactes (C1-5), (C1-13) et (C1-22) peuvent être réécrites sous la forme:

$$
\begin{aligned}
\frac{\partial N}{\partial t}+\nabla \cdot \mathbf{J} & =0 \\
m N\left(\frac{\partial}{\partial t}+\mathbf{V} \cdot \nabla\right) \mathbf{V}+\nabla P^{(0)} & =-\nabla \cdot\left(\overline{\mathbf{P}}-P^{(0)} \mathbf{1}\right) \\
\frac{\partial W}{\partial t}+\nabla \cdot(W \mathbf{V})+P^{(0)} \nabla \cdot \mathbf{V} & =-\nabla \cdot \mathbf{J}_{T}-\bar{\Lambda}:\left(\overline{\mathbf{P}}-P^{(0)} \mathbf{1}\right)
\end{aligned}
$$

où $P^{(0)}$ est la variable lente définie par (cf. (46)):

$$
P^{(0)}=N_{L}^{(0)} k_{B} T^{(0)}\left[1+\frac{1}{2} N_{L}^{(0)} \Sigma_{0}\left(T^{(0)}\right)\right]
$$

Dans le membre de gauche, toutes les variables sont des variables hydrodynamiques; dans celui de droite, à part le tenseur $\overline{\boldsymbol{\Lambda}}$ des gradients de la vitesse qui est une variable lente, toutes les variables sont rapides. Ces équations nous permettent de vérifier que les variables hydrodynamiques ont une variation temporelle qui s'annule pour une situation uniforme dans l'espace (ce qui est faux pour les variables rapides) et sont donc bien des variables lentes.

Quant à l'évolution des variables rapides, elle peut être obtenue à partir de (64) et de l'équation cinétique ; on obtient ainsi:

$$
D_{\mathbf{p}} g^{r a p}=-D_{\mathbf{p}} g^{(0)}+I_{B}\left[f^{(0)}+f^{r a p}\right]+K_{\text {coll }}\left[f^{(0)}+f^{r a p}\right]+R_{\Xi}\left[f^{(0)}+f^{r a p}\right]
$$

où $D_{p}$ est l'opérateur défini par (3). En l'absence de gradients spatiaux, (69) se réduit à:

$$
\frac{\partial}{\partial t} g^{r a p}=I_{B}\left[f^{(0)}+f^{r a p}\right]
$$

La discussion de l'évolution de $g^{r a p}$ est alors celle qui a été menée au $\S 2.1$, qui montre que la présence de l'intégrale de collision de Boltzmann $I_{B}$ conduit à une annulation rapide de la partie non gaussienne de $f, f^{r a p}$, et de $g^{r a p}$. Dans l'esprit de la méthode de Chapman Enskog, nous allons supposer que les variations spatiales des grandeurs hydrodynamiques sont suffisamment lentes pour que $f^{r a p}$, c'est à dire l'ensemble des variables rapides, reste toujours très petit devant $f^{(0)}$. 
Mathématiquement, en supposant qu'à un instant $t$ donné $g^{r a p}$ est nul, on voit en utilisant (69), que $g^{r a p}$ possède deux "termes source" qui le régénèrent par couplage aux variables lentes; le premier est:

$$
-D_{\mathbf{p}} g^{(0)}(\mathbf{p})
$$

où les variations de $f^{(0)}$ proviennent implicitement de celles des grandeurs hydrodynamiques contenues dans sa définition (63), ces variations étant d'ailleurs elles mêmes données par (67); comme l'intégrale de collision de Boltzmann est nulle pour la fonction $f^{(0)}$, l'autre source de couplage provient du terme:

$$
K_{\text {coll }}\left[f^{(0)}(\mathbf{p})\right]+R_{\Xi}\left[f^{(0)}(\mathbf{p})\right]
$$

où, à nouveau, on peut exprimer les variations spatiales de $f^{(0)}$ en fonction des grandeurs hydrodynamiques.

Globalement, si nous supposons comme Chapman et Enskog que les variations spatiales de toutes les grandeurs hydrodynamiques se font sur des distances très grandes à l'échelle du libre parcours moyen dans le gaz, ces termes source ne sont pas suffisants pour combattre efficacement l'amortissement des variables rapides induit par l'intégrale de collision de Boltzmann, et la fonction $g^{r a p}$ (et avec elle $f^{r a p}$ ) reste très petite. La méthode à suivre est alors claire: on va calculer les valeurs de $g^{r a p}$ par un développement en série de puissances du rapport entre "termes source" et amortissement de Boltzmann des variables rapides, c'est à dire entre le libre parcours moyen et une distance caractéristique des variations spatiales de $N, \mathbf{V}$ et $W$. Nous commençons dans le paragraphe suivant par l'ordre zéro de ce développement.

\section{HYDRODYNAMIQUE NON DISSIPATIVE}

A l'ordre zéro, les équations dynamiques se réduisent aux équations (67) sans second membre:

$$
\begin{aligned}
D_{h} N & =-N \nabla \cdot \mathbf{V} \\
m N D_{h} \mathbf{V} & =-\nabla P^{(0)} \\
D_{h}\left(\frac{W N_{L}}{W_{L} N} k_{B} T^{(0)}\right) & =-\frac{2 P^{(0)}}{3 N} \nabla \cdot \mathbf{V}
\end{aligned}
$$

Dans ces équations, la "dérivée hydrodynamique" $D_{h}$ est donnée par:

$$
D_{h}=\frac{\partial}{\partial t}+\mathbf{V} \cdot \nabla
$$

A l'ordre zéro où nous travaillons, les grandeurs calculées à partir de $f$ et de $f^{(0)}$ sont confondues, et nous omettrons de les différencier. Nous nous limiterons en outre aux plus basses corrections en densité, si bien qu'à partir de (61) et (68) nous écrirons:

$$
\begin{aligned}
P & =N k_{B} T\left(1-N \Sigma_{0} / 2\right) \\
\frac{W N_{L}}{W_{L} N} & =1-N \Sigma_{0} / 2+N \Sigma_{1} / 3
\end{aligned}
$$

Si l'on élimine $\nabla \cdot \mathbf{V}$ entre la première et la dernière équation de (73), il vient:

$$
D_{h}\left[\left(1-N \Sigma_{0} / 2+N \Sigma_{1} / 3\right) k_{B} T\right]=\frac{2 k_{B} T}{3 N}\left(1-N \Sigma_{0} / 2\right) D_{h}[N]
$$

soit:

$$
\left(1-N \Sigma_{0} / 2+N \Sigma_{1} / 3\right) D_{h} \log \left[\left(1-N \Sigma_{0} / 2+N \Sigma_{1} / 3\right) k_{B} T\right]=\frac{2}{3}\left(1-N \Sigma_{0} / 2\right) D_{h} \log [N]
$$


Lorsque l'on néglige, comme dans la théorie de Boltzmann, les corrections $N \Sigma_{0,1}=0$, on obtientpour l'équation d'état du système (à l'ordre zéro en densité):

$$
T N^{-3 / 2}=\text { Constante }
$$

qui, à l'ordre un en densité, devient:

$$
F(N, T)=\text { Constante }
$$

où les dérivées partielles de $F$ apparaissent, à un facteur intégrant près, dans les coefficients des dérivées logarithmiques de (77):

$$
-\frac{\partial F}{\partial N}\left(\frac{\partial F}{\partial T}\right)^{-1}=\frac{\partial T}{\partial N} \simeq \frac{2 T}{3 N}\left(1-N \Sigma_{1} / 3\right)
$$

Dans les deux cas, l'équation d'état correspond à des transformations adiabatiques puisque le courant de chaleur reste constamment nul. De cette expression on peut tirer la compressibilité isentropique:

$$
\chi^{-1}=N \frac{\partial P}{\partial N} \simeq \frac{5}{3} N k_{B} T\left(1-N \Sigma_{0} / 2-2 N \Sigma_{1} / 15\right)
$$

On peut supposer que les variables hydrodynamiques sont proches de valeurs à l'équilibre $N_{e}, \mathbf{V}_{e}$ et $T_{e}$ :

$$
\begin{aligned}
N & =N_{e}+n \\
\mathbf{V} & =\mathbf{v} \\
k_{B} T & =k_{B} T_{e}+\theta
\end{aligned}
$$

et linéariser par rapport aux variations des variables $n$, $\mathbf{v}$, et $\theta$; on obtient alors:

$$
\begin{aligned}
\frac{\partial}{\partial t} n & =-N_{e} \nabla \cdot \mathbf{V} \\
m N_{e} \frac{\partial}{\partial t} \mathbf{v} & =-\chi^{-1} N_{e}^{-1} \nabla n
\end{aligned}
$$

De là on peut déduire l'équation de Helmholtz:

$$
\frac{\partial^{2}}{\partial t^{2}} n=c^{2} \Delta n
$$

où le carré de la vitesse du son est donné par:

$$
c^{2}=\left(m N_{e} \chi\right)^{-1}
$$

A l'ordre zéro, la propagation des ondes sonores est donc non dispersive.

\section{HYDRODYNAMIQUE VISQUEUSE}

Nous allons maintenant un degré plus loin dans le développement en gradients des grandeurs hydrodynamiques, c'est à dire que nous utiliserons la forme (69) de l'équation cinétique pour déterminer, à l'ordre le plus bas, la partie non gaussienne $f^{r a p}$ de $f$, ainsi que $g^{\text {rap }}$. Notant $f^{(1)}$ 
et $g^{(1)}$ ces termes linéaires dans le développement en puissances des gradients spatiaux, l'équation cinétique (69) devient:

$$
D_{\mathbf{p}} g^{(1)}=-D_{\mathbf{p}} g^{(0)}+I_{B}^{l}\left[f^{(0)}, f^{(1)}\right]+K_{\text {coll }}\left[f^{(0)}\right]+R_{\Xi}\left[f^{(0)}\right]
$$

où l'opérateur de collision linéarisé $I_{B}^{l}$ est donné par:

$$
\begin{aligned}
I_{B}^{l}\left[f^{(0)}, f^{(1)}\right](\mathbf{p})=\int d^{3} q \int d^{2} \hat{q}^{\prime} & q \sigma_{k}\left(\hat{q} \cdot \hat{q}^{\prime}\right)\left\{f^{(0)}\left(\mathbf{2}^{\prime}\right) f^{(1)}\left(\mathbf{1}^{\prime}\right)+f^{(0)}\left(\mathbf{1}^{\prime}\right) f^{(1)}\left(\mathbf{2}^{\prime}\right)\right. \\
& \left.-f^{(0)}(\mathbf{p}-\mathbf{q}) f^{(1)}(\mathbf{q})-f^{(0)}(\mathbf{q}) f^{(1)}(\mathbf{p}-\mathbf{q})\right\}(86-b)
\end{aligned}
$$

(on note ici encore $\mathbf{1}^{\prime}$ et $\mathbf{2}^{\prime}$ les impulsions $\mathbf{p}-\mathbf{q} / 2+\mathbf{q}^{\prime} / 2$ et $\mathbf{p}-\mathbf{q} / 2-\mathbf{q}^{\prime} / 2$ ). A l'ordre un, $g^{(1)}$ est donné par la linéarisation des équations (2):

$$
g^{(1)}(\mathbf{p})=f^{(1)}(\mathbf{p})+\int d^{3} q \Xi(q)\left[f^{(0)}(\mathbf{p}) f^{(1)}(\mathbf{p}-\mathbf{q})+f^{(1)}(\mathbf{p}) f^{(0)}(\mathbf{p}-\mathbf{q})\right]
$$

Seuls les ordres zéro des termes en $K_{\text {coll }}$ et $R_{\Xi}$ ont été gardés dans (86-a), puisque leur définition comporte des gradients spatiaux. En outre, dans le membre de gauche de (86-a), le gradient spatial de $g^{(1)}$ pourra être négligé (ce serait un terme du deuxième ordre en gradients), ce qui fait que la partie linéaire de la réponse stationnaire de la fonction de distribution à l'application de gradients de densité, vitesse et température est donnée par:

$$
I_{B}^{l}\left[f^{(0)}, f^{(1)}\right]=D_{\mathbf{p}} g^{(0)}-K_{\text {coll }}\left[f^{(0)}\right]-R_{\Xi}\left[f^{(0)}\right]
$$

Enfin, $f^{(1)}\left(\right.$ et $\left.g^{(1)}\right)$ seront entièrement déterminés par la nécessité de vérifier les trois contraintes (65), qui donnent:

$$
\int d^{3} p F(\mathbf{p}) g^{(1)}(\mathbf{p})=0 \quad \text { pour } F(\mathbf{p})=1, \mathbf{p} \text { ou } p^{2}
$$

Un certain nombre de différences apparaissent clairement par rapport au traitement habituel en théorie de Boltzmann:

- le terme source (membre de droite) contient des corrections en densité à sa valeur usuelle $D_{\mathbf{p}} f^{(0)}$, à la fois dans le terme source modifié $\left(D_{\mathbf{p}} g^{(0)}\right)$ et dans le terme source supplémentaire $\left(-K_{\text {coll }}\left[f^{(0)}\right]-R_{\Xi}\left[f^{(0)}\right]\right)$; densité.

- les contraintes (sur $f$ ) diffèrent, elles aussi, de leur valeur habituelle, par des corrections de

Par contre, le terme d'amortissement donné par l'opérateur de collision linéarisé est le même qu'en théorie de Boltzmann, et ne contient aucune correction de densité. Ce résultat est simplement la conséquence du fait que seules les collisions binaires sont envisagées dans le cadre de tout ce travail, et nous y reviendrons en conclusion de ce $\S 5$.

Nous allons maintenant expliciter ces différents termes, ce qui nous permettra de calculer les coefficients de transport pour un gaz modérément dense.

\subsection{TERME SOURCE}

Le terme source supplémentaire fait intervenir le gradient de $f^{(0)}$, pour diverses valeurs de l'impulsion. Pour une impulsion quelconque $\mathbf{p}^{\prime}$, ce gradient est donné par:

$$
\nabla f^{(0)}\left(\mathbf{p}^{\prime}\right)=f^{(0)}\left(\mathbf{p}^{\prime}\right)\left\{\frac{\nabla N_{L}^{(0)}}{N_{L}^{(0)}}+\sum_{i} \frac{p_{i}^{\prime}-m V_{i}}{k_{B} T^{(0)}} \nabla V_{i}+\left(\frac{\left(\mathbf{p}^{\prime}-m \mathbf{V}\right)^{2}}{2 m k_{B} T^{(0)}}-\frac{3}{2}\right) \frac{\nabla T^{(0)}}{T^{(0)}}\right\}
$$


En (62), nous avions défini $N_{L}^{(0)}$ et $T^{(0)}$ en termes de densité de particules et d'énergie, mais il est ici plus commode de remplacer la donnée de $W$ par celle de la température: par exemple, on désire trouver que le courant de chaleur est proportionnel au gradient de $T$ et non à une combinaison de gradients de $N$ et de $W$. On obtient alors:

$$
\begin{aligned}
\nabla f^{(0)}\left(\mathbf{p}^{\prime}\right)=f^{(0)}\left(\mathbf{p}^{\prime}\right) & \left\{\frac{\partial N_{L}^{(0)}}{\partial N} \frac{\nabla N}{N_{L}^{(0)}}+\sum_{i} \frac{p_{i}^{\prime}-m V_{i}}{k_{B} T^{(0)}} \nabla V_{i}\right. \\
& \left.+\left(\frac{\left(\mathbf{p}^{\prime}-m \mathbf{V}\right)^{2}}{2 m k_{B} T^{(0)}}-\frac{3}{2}+\frac{T^{(0)}}{N_{L}^{(0)}} \frac{\partial N_{L}^{(0)}}{\partial T^{(0)}}\right) \frac{\nabla T^{(0)}}{T^{(0)}}\right\}
\end{aligned}
$$

où les dérivées partielles de $N_{L}^{(0)}$ seront explicitées plus loin (formule (99-a)).

La partie modifiée du terme source a, d'après la définition $(2)$ de $g^{(0)}$, pour expression:

$$
D_{\mathbf{p}} g^{(0)}(\mathbf{p})=\frac{g^{(0)}(\mathbf{p})}{f^{(0)}(\mathbf{p})} D_{\mathbf{p}} f^{(0)}(\mathbf{p})+f^{(0)}(\mathbf{p}) \int d^{3} q \Xi(q) D_{\mathbf{p}} f^{(0)}(\mathbf{p}-\mathbf{q})
$$

Il nous faut donc, à partir de la définition (63), calculer l'expression de l'action de l'opérateur $D_{\mathbf{p}}$ sur $f^{(0)}$ pour une valeur $\mathbf{p}^{\prime}$ de l'impulsion (on l'utilisera pour $\mathbf{p}^{\prime}=\mathbf{p}$ ou $\mathbf{p}-\mathbf{q}$ ). Il s'agit d'un calcul classique en théorie de Boltzmann, qui conduit au résultat:

$$
D_{\mathbf{p}} f^{(0)}\left(\mathbf{p}^{\prime}\right)=f^{(0)}\left(\mathbf{p}^{\prime}\right)\left\{\frac{D_{\mathbf{p}} N_{L}^{(0)}}{N_{L}^{(0)}}+\frac{\mathbf{p}^{\prime}-m \mathbf{V}}{k_{B} T^{(0)}} \cdot D_{\mathbf{p}} \mathbf{V}+\left(\frac{\left(\mathbf{p}^{\prime}-m \mathbf{V}\right)^{2}}{2 m k_{B} T^{(0)}}-\frac{3}{2}\right) \frac{D_{\mathbf{p}} T^{(0)}}{T^{(0)}}\right\}
$$

qui, pour les mêmes raisons que plus haut, sera plutôt écrit:

$$
\begin{aligned}
D_{\mathbf{p}} f^{(0)}\left(\mathbf{p}^{\prime}\right)=f^{(0)}\left(\mathbf{p}^{\prime}\right)\{ & \frac{\partial N_{L}^{(0)}}{\partial N} \frac{D_{\mathbf{p}} N}{N_{L}^{(0)}}+\frac{\mathbf{p}^{\prime}-m \mathbf{V}}{k_{B} T^{(0)}} \cdot D_{\mathbf{p}} \mathbf{V} \\
& \left.+\left(\frac{\left(\mathbf{p}^{\prime}-m \mathbf{V}\right)^{2}}{2 m k_{B} T^{(0)}}-\frac{3}{2}+\frac{T^{(0)}}{N_{L}^{(0)}} \frac{\partial N_{L}^{(0)}}{\partial T^{(0)}}\right) \frac{D_{\mathbf{p}} T^{(0)}}{T^{(0)}}\right\}
\end{aligned}
$$

Les termes $D_{\mathbf{p}} N, D_{\mathbf{p}} \mathbf{V}$ et $D_{\mathbf{p}} T^{(0)}$ sont à calculer à l'ordre zéro, par exemple à partir des équations hydrodynamiques (73) pour les deux premiers:

$$
\begin{aligned}
& D_{\mathbf{p}} N=\left(\frac{\mathbf{p}}{m}-\mathbf{V}\right) \cdot \nabla N-N \nabla \cdot \mathbf{V} \\
& D_{\mathbf{p}} \mathbf{V}=\left[\left(\frac{\mathbf{p}}{m}-\mathbf{V}\right) \cdot \nabla\right] \mathbf{V}-\frac{1}{m N}\left\{\frac{\partial P^{(0)}}{\partial N_{L}^{(0)}} \frac{\partial N_{L}^{(0)}}{\partial N} \nabla N+\left(\frac{\partial P^{(0)}}{\partial N_{L}^{(0)}} \frac{\partial N_{L}^{(0)}}{\partial T^{(0)}}+\frac{\partial P^{(0)}}{\partial T^{(0)}}\right) \nabla T^{(0)}\right\}
\end{aligned}
$$

où l'accolade du second membre résulte de l'écriture explicite du gradient de la pression. Quant à $D_{\mathbf{P}} T^{(0)}$, il peut être tiré de:

$$
D_{\mathbf{p}} W=\frac{\partial W}{\partial N} D_{\mathbf{p}} N+\frac{\partial W}{\partial T^{(0)}} D_{\mathbf{p}} T^{(0)}
$$

Utilisant (67) et le fait qu'à l'ordre zéro $\mathbf{J}_{T}$ est nul et $\overline{\mathbf{P}}$ est scalaire, on obtient:

$$
D_{\mathbf{p}} T^{(0)}=\left(\frac{\mathbf{p}}{m}-\mathbf{V}\right) \cdot \nabla T^{(0)}+\left(\frac{\partial W}{\partial T^{(0)}}\right)^{-1} \times\left(N \frac{\partial W}{\partial N}-W-P^{(0)}\right) \times \nabla \cdot \mathbf{V}
$$


L'utilisation des formules (95), (96) et (98) dans l'expression du terme source modifié, et l'usage de l'équation (91) nous permettrons dans les paragraphes qui suivent d'exprimer complètement le terme source de l'équation cinétique linéarisée en fonction des gradients des grandeurs physiques significatives: densité, vitesse et température. Un certain nombre de dérivées partielles figurent dans les formules ci-dessus, dont nous donnons les expressions simplement tirées de la définition des fonctions qu'elles font intervenir:

$$
\begin{aligned}
& \left.\frac{\partial N_{L}^{(0)}}{\partial N}\right|_{T^{(0)}}=\frac{1}{1+2 N_{L}^{(0)} \Sigma_{0}} \quad \text { et }\left.\quad \frac{\partial N_{L}^{(0)}}{\partial T^{(0)}}\right|_{N}=-\frac{N_{L}^{(0)}}{T^{(0)}} \frac{N_{L}^{(0)} \Sigma_{1}-3 N_{L}^{(0)} \Sigma_{0} / 2}{1+2 N_{L}^{(0)} \Sigma_{0}} \quad(99-a) \\
& \left.\frac{\partial P^{(0)}}{\partial N_{L}^{(0)}}\right|_{T^{(0)}}=\frac{N}{N_{L}^{(0)}} k_{B} T^{(0)} \quad \text { et }\left.\quad \frac{\partial P^{(0)}}{\partial T^{(0)}}\right|_{N_{L}^{(0)}}=\frac{P^{(0)}}{T^{(0)}}+k_{B} N_{L}^{(0)}\left(\frac{N_{L}^{(0)} \Sigma_{1}}{2}-\frac{3 N_{L}^{(0)} \Sigma_{0}}{4}\right)(99-b) \\
& \left.\frac{\partial W}{\partial T^{(0)}}\right|_{N}=\frac{3}{2} N k_{B}\left(\alpha+T^{(0)} \frac{\partial \alpha}{\partial T^{(0)}}\right) \quad \text { et }\left.\quad \frac{\partial W}{\partial N}\right|_{T^{(0)}}=\frac{W}{N}+\frac{3}{2} N k_{B} T^{(0)} \frac{\partial \alpha}{\partial N} \quad(99-c) \\
& \text { avec } \quad \alpha=1+\frac{N_{L}^{(0)}}{N}\left(\frac{N_{L}^{(0)} \Sigma_{1}}{3}-\frac{N_{L}^{(0)} \Sigma_{0}}{2}\right)
\end{aligned}
$$

Rappelons ici l'expression de $G_{\text {coll }}^{\xi}$ et $G_{\text {coll }}^{\Delta}$ dont $K_{\text {coll }}$ est la somme:

$$
\begin{aligned}
G_{\text {coll }}^{\xi}\left[f^{(0)}(\mathbf{p})\right] & =\frac{1}{m} \int d^{3} q \xi_{F}(q) \mathbf{q} \cdot\left\{f^{(0)}(\mathbf{p}) \nabla f^{(0)}(\mathbf{p}-\mathbf{q})-f^{(0)}(\mathbf{p}-\mathbf{q}) \nabla f^{(0)}(\mathbf{p})\right\} \\
+ & \frac{1}{m} \int d^{3} q \int d^{2} \hat{q}^{\prime} \xi_{k}(q, \theta) \mathbf{q}^{\prime} \cdot\left\{f^{(0)}\left(\mathbf{2}^{\prime}\right) \nabla f^{(0)}\left(\mathbf{1}^{\prime}\right)-f^{(0)}\left(\mathbf{1}^{\prime}\right) \nabla f^{(0)}\left(\mathbf{2}^{\prime}\right)\right\} \\
G_{\text {coll }}^{\Delta}\left[f^{(0)}(\mathbf{p})\right] & \left.=\frac{1}{m} \int d^{3} q q \Delta_{F}(q)\left\{\nabla_{\mathbf{p}}^{\perp} f^{(0)}(\mathbf{p}) \cdot \nabla f^{(0)}(\mathbf{p}-\mathbf{q})-f^{(0)}(\mathbf{p}) \nabla\right)_{\mathbf{p}}^{\perp} \nabla f^{(0)}(\mathbf{p}-\mathbf{q})\right\} \\
+ & \frac{1}{m} \int d^{3} q \int d^{2} \hat{q}^{\prime} \Delta_{k}(q, \theta) \mathbf{q}^{\perp} \cdot\left\{f^{(0)}\left(\mathbf{2}^{\prime}\right) \nabla f^{(0)}\left(\mathbf{1}^{\prime}\right)-f^{(0)}\left(\mathbf{1}^{\prime}\right) \nabla f^{(0)}\left(\mathbf{2}^{\prime}\right)\right\}
\end{aligned}
$$

Dans l'expression de $G_{\text {coll }}^{\Delta}$, nous avons omis d'écrire l'intégrale:

$$
\frac{1}{m} \int d^{3} q \int d^{2} \hat{q}^{\prime} \Delta_{k}(q, \theta) \mathbf{q}^{\prime \perp} \cdot \nabla\left\{f^{(0)}\left(\mathbf{1}^{\prime}\right) f^{(0)}\left(\mathbf{2}^{\prime}\right)\right\}
$$

Celle-ci est en effet nulle, comme le montrent l'utilisation de:

$$
f^{(0)}\left(\mathbf{1}^{\prime}\right) f^{(0)}\left(\mathbf{2}^{\prime}\right)=f^{(0)}(\mathbf{p}) f^{(0)}(\mathbf{p}-\mathbf{q})
$$

et une intégration de $\hat{q}^{\prime}$ autour de $\hat{q}$.

Examinons maintenant la contribution de chacun des différents gradients, en supposant à chaque fois les autres nuls.

\subsubsection{TERMES EN $\nabla N$}


Commençons par montrer que le terme en $K_{\text {coll }}$ ne contribue pas. Puisque le terme en $\nabla N$ dans $\nabla f^{(0)}\left(\mathbf{p}^{\prime}\right)$ est simplement proportionnel à $f^{(0)}\left(\mathbf{p}^{\prime}\right)$, le terme issu de $G_{\text {coll }}^{\xi}$ est nul, ainsi que le terme en $\Delta_{k}$ de $G_{\text {coll }}^{\Delta}$. Le terme en $\Delta_{F}$ est nul lui aussi car $\nabla_{\mathbf{p}} f^{(0)}\left(\mathbf{p}^{\prime}\right)$ est proportionnel à $\mathbf{p}^{\prime} f^{(0)}\left(\mathbf{p}^{\prime}\right)$, et les projections perpendiculaires (à $\left.\mathbf{q}\right)$ de $\mathbf{p}$ et $\mathbf{p}-\mathbf{q}$ sont égales.

La contribution du terme source modifié s'exprime, en substituant (95) et (96) dans (94) pour $\mathbf{V}$ et $T^{(0)}$ uniformes:

$$
\begin{aligned}
& D_{\mathbf{p}} g^{(0)}(\mathbf{p})=g^{(0)}(\mathbf{p}) A_{N}(\mathbf{p}) \\
& \quad+f^{(0)}(\mathbf{p}) \int d^{3} q \Xi(q) f^{(0)}(\mathbf{p}-\mathbf{q})\left\{A_{N}(\mathbf{p})+\frac{\mathbf{q} \cdot \nabla N}{N k_{B} T^{(0)}} \frac{\partial P^{(0)}}{\partial N_{L}^{(0)}} \frac{\partial N_{L}^{(0)}}{\partial N}\right\}
\end{aligned}
$$

où on a posé:

$$
A_{N}(\mathbf{p})=\left(\frac{\mathbf{p}}{m}-\mathbf{V}\right) \cdot \nabla N\left\{\frac{1}{N_{L}^{(0)}} \frac{\partial N_{L}^{(0)}}{\partial N}-\frac{1}{N k_{B} T^{(0)}} \frac{\partial P^{(0)}}{\partial N_{L}^{(0)}} \frac{\partial N_{L}^{(0)}}{\partial N}\right\}
$$

Utilisant (99-b), on montre que $A_{N}(\mathbf{p})$ est nul et il vient:

$$
D_{\mathbf{p}} g^{(0)}(\mathbf{p})=\frac{1}{N_{L}^{(0)}} \frac{\partial N_{L}^{(0)}}{\partial N} f^{(0)}(\mathbf{p}) \int d^{3} q \Xi(q) \mathbf{q} \cdot \nabla N f^{(0)}(\mathbf{p}-\mathbf{q})
$$

Cette expression est la même que celle du terme en $R_{\Xi}$ obtenue en utilisant (91), si bien qu'au total le terme source ne dépend pas du gradient de la densité.

Il y a là un point qui n'est pas fortuit, mais joue un rôle important dans la cohérence de la théorie: la présence d'un terme en $\mathbf{p} \cdot \nabla N$, vectoriel vis à vis des variables angulaires de $\mathbf{p}$, exciterait le courant de chaleur comme le fait le terme en $\mathbf{p} \cdot \nabla T$ calculé au paragraphe 5.1.3. Le fait que nous trouverons un courant de chaleur qui ne dépend que du gradient de la température est un contrôle de la pertinence de la définition de $T$ que nous avons choisie plus haut. On peut remarquer que la seconde correction du viriel à la pression joue un rôle essentiel dans l'annulation de (104).

\subsubsection{TERMES EN $\bar{\Lambda}$}

Supposons maintenant $N$ et $T^{(0)}$ uniformes; chacun des termes dans l'expression (91) de $\nabla f^{(0)}$ introduit une contribution au terme source modifié. Celles résultant de la substitution de (95) et (98) sont proportionnelles à $\nabla \cdot \mathbf{V}$, tandis que celle issue de la substitution de (96) peut être décomposée en:

$$
\begin{aligned}
\frac{\mathbf{p}^{\prime}-m \mathbf{V}}{k_{B} T^{(0)}} \cdot D_{\mathbf{p}} \mathbf{V}= & \frac{1}{m k_{B} T^{(0)}}\left[\left(\mathbf{p}^{\prime}-m \mathbf{V}\right) \otimes\left(\mathbf{p}^{\prime}-m \mathbf{V}\right)\right]: \breve{\Lambda}+\frac{1}{3 m k_{B} T^{(0)}}\left(\mathbf{p}^{\prime}-m \mathbf{V}\right)^{2} \nabla \cdot \mathbf{V} \\
& +\frac{\mathbf{p}-\mathbf{p}^{\prime}}{m} \cdot \sum_{i} \frac{p_{i}^{\prime}-m V_{i}}{k_{B} T^{(0)}} \nabla V_{i}
\end{aligned}
$$

où on a noté $\breve{\Lambda}$ la partie sans trace de $\bar{\Lambda}$ :

$$
\breve{\Lambda}=\bar{\Lambda}-\frac{1}{3} \nabla \cdot \mathbf{V} \overline{\mathbf{1}}
$$

Introduisons les expressions suivantes:

$$
A_{\breve{\Lambda}}\left(\mathbf{p}^{\prime}\right)=\frac{1}{m k_{B} T^{(0)}}\left[\left(\mathbf{p}^{\prime}-m \mathbf{V}\right) \otimes\left(\mathbf{p}^{\prime}-m \mathbf{V}\right)\right]: \breve{\Lambda}
$$




$$
\begin{aligned}
A_{\nabla \cdot \mathbf{V}}\left(\mathbf{p}^{\prime}\right) & =\left\{\frac{\left(\mathbf{p}^{\prime}-m \mathbf{V}\right)^{2}}{3 m k_{B} T^{(0)}}\left[1+\frac{3}{2 T^{(0)}}\left(\frac{\partial W}{\partial T^{(0)}}\right)^{-1}\left(N \frac{\partial W}{\partial N}-W-P^{(0)}\right)\right]\right. \\
& \left.+\left(\frac{1}{N_{L}^{(0)}} \frac{\partial N_{L}^{(0)}}{\partial T^{(0)}}-\frac{3}{2 T^{(0)}}\right) \times\left(\frac{\partial W}{\partial T^{(0)}}\right)^{-1}\left(N \frac{\partial W}{\partial N}-W-P^{(0)}\right)-\frac{N}{N_{L}^{(0)}} \frac{\partial N_{L}^{(0)}}{\partial N}\right\} \nabla \cdot \mathbf{V}
\end{aligned}
$$

On peut alors écrire le terme source modifié sous la forme:

$$
\begin{aligned}
D_{\mathbf{p}} g^{(0)}(\mathbf{p}) & =g^{(0)}(\mathbf{p})\left[A_{\nabla \cdot \mathbf{v}}(\mathbf{p})+A_{\breve{\Lambda}}(\mathbf{p})\right] \\
& +f^{(0)}(\mathbf{p}) \int d^{3} q \Xi(q) f^{(0)}(\mathbf{p}-\mathbf{q})\left[A_{\nabla \cdot \mathbf{v}}(\mathbf{p}-\mathbf{q})+A_{\breve{\Lambda}}(\mathbf{p}-\mathbf{q})\right] \\
& +R_{\Xi}\left[f^{(0)}(\mathbf{p})\right]
\end{aligned}
$$

Le terme $R_{\Xi}$ est reconstruit par la contribution du troisième terme de (106) lorsque $\mathbf{p}^{\prime}=\mathbf{p}-\mathbf{q}$.

Le calcul des coefficients entrant dans la définition de $A_{\nabla \cdot \mathbf{v}}$ peut être fait à partir des formules (99), et nous en présentons le résultat limité à l'ordre un en densité (première correction du viriel):

$$
A_{\nabla \cdot \mathbf{V}}\left(\mathbf{p}^{\prime}\right)=\left\{\frac{\left(\mathbf{p}^{\prime}-m \mathbf{V}\right)^{2}}{3 m k_{B} T^{(0)}}\left(\frac{N_{L}^{(0)} \Sigma_{2}}{3}-\frac{N_{L}^{(0)} \Sigma_{1}}{2}\right)+\left(\frac{7 N_{L}^{(0)} \Sigma_{1}}{6}-\frac{N_{L}^{(0)} \Sigma_{2}}{3}\right)\right\} \nabla \cdot \mathbf{V}
$$

On retrouve un résultat classique en théorie de Boltzmann: à l'ordre zéro en densité, il n'y a pas de terme source proportionnel à la divergence de la vitesse, et seule la viscosité de cisaillement est présente dans un gaz parfait. A l'ordre un en densité nous trouvons cependant un terme source non nul, laissant présager l'existence d'une seconde viscosité dans un gaz modérément dense. A cet ordre, l'ensemble du terme source s'écrit:

$$
\begin{aligned}
D_{\mathbf{p}} g^{(0)}(\mathbf{p})-R_{\Xi}\left[f^{(0)}(\mathbf{p})\right]-K_{\text {coll }}\left[f^{(0)}(\mathbf{p})\right] & =g^{(0)}(\mathbf{p})\left[A_{\nabla \cdot \mathbf{V}}(\mathbf{p})+A_{\breve{\Lambda}}(\mathbf{p})\right] \\
& +f^{(0)}(\mathbf{p}) \int d^{3} q \Xi(q) f^{(0)}(\mathbf{p}-\mathbf{q}) A_{\breve{\Lambda}}(\mathbf{p}-\mathbf{q}) \\
& -K_{\text {coll }}\left[f^{(0)}(\mathbf{p})\right]
\end{aligned}
$$

Pour estimer enfin la contribution du terme $K_{\text {coll }}$, nous aurons besoin par la suite de l'expression du gradient de $f^{(0)}$; la formule (91) peut être écrite:

$$
\nabla f^{(0)}\left(\mathbf{p}^{\prime}\right)=\frac{1}{k_{B} T^{(0)}} f^{(0)}\left(\mathbf{p}^{\prime}\right)\left(\mathbf{p}^{\prime}-m \mathbf{V}\right) \cdot \overline{\boldsymbol{\Gamma}}_{\mathbf{V}}
$$

où $\overline{\boldsymbol{\Gamma}}_{\mathbf{V}}$ est le tenseur des gradients de la vitesse, dont $\bar{\Lambda}$ est la partie symétrique.

\subsubsection{TERMES EN $\nabla T$}

Estimons d'abord la contribution du terme source modifié en substituant (96) et (98) dans (94) pour $N$ et $\mathbf{V}$ uniformes:

$$
\begin{aligned}
D_{\mathbf{p}} g^{(0)}(\mathbf{p}) & =g^{(0)}(\mathbf{p}) A_{T}(\mathbf{p}) \\
& +f^{(0)}(\mathbf{p}) \int d^{3} q \Xi(q) f^{(0)}(\mathbf{p}-\mathbf{q}) A_{T}(\mathbf{p}-\mathbf{q})+R_{\Xi}\left[f^{(0)}(\mathbf{p})\right]
\end{aligned}
$$


où on a posé:

$$
A_{T}\left(\mathbf{p}^{\prime}\right)=\left(\frac{\mathbf{p}^{\prime}}{m}-\mathbf{V}\right) \cdot \frac{\nabla T^{(0)}}{T^{(0)}}\left\{\frac{\left(\mathbf{p}^{\prime}-m \mathbf{V}\right)^{2}}{2 m k_{B} T^{(0)}}-\frac{5}{2}+\frac{N_{L}^{(0)}}{2 N}\left(\frac{5}{2} N_{L}^{(0)} \Sigma_{0}-N_{L}^{(0)} \Sigma_{1}\right)\right\}
$$

La reconstruction d'un terme égal à $R_{\Xi}$ résulte de la dépendance en $\mathbf{p} \cdot \nabla T^{(0)}$ du terme en $D_{\mathbf{p}} T^{(0)}$ dans $D_{\mathbf{p}} f^{(0)}(\mathbf{p}-\mathbf{q})$, alors que celle de $A_{T}(\mathbf{p}-\mathbf{q})$ est en $(\mathbf{p}-\mathbf{q}) \cdot \nabla T^{(0)}$.

Pour le calcul de $K_{\text {coll }}$, les termes simplement proportionnels à $f^{(0)}\left(\mathbf{p}^{\prime}\right)$ ne contribuent pas pour les raisons exposées au $\S$ 5.1.1. Introduisons, afin d'obtenir des expressions formellement proches de celles relatives au terme en $\breve{\Lambda}$, le tenseur $\overline{\boldsymbol{\Gamma}}_{T}$ défini par:

$$
\overline{\boldsymbol{\Gamma}}_{T}=\left(\frac{\mathbf{p}}{m}-\mathbf{V}\right) \otimes \frac{\nabla T^{(0)}}{T^{(0)}}
$$

Avec cette notation, la partie restante de $\nabla f^{(0)}\left(\mathbf{p}^{\prime}\right)$, proportionnelle à la densité d'énergie pour l'impulsion $\mathbf{p}^{\prime}$, s'écrit:

$$
\nabla f^{(0)}\left(\mathbf{p}^{\prime}\right)=\frac{1}{k_{B} T^{(0)}} f^{(0)}\left(\mathbf{p}^{\prime}\right)\left\{\left(\mathbf{p}^{\prime}-m \mathbf{V}\right) \cdot \overline{\boldsymbol{\Gamma}}_{T}+\frac{\left(\mathbf{p}^{\prime}-\mathbf{p}\right)^{2}}{2 m} \frac{\nabla T^{(0)}}{T^{(0)}}-\frac{1}{2}(\mathbf{p}-m \mathbf{V}) \cdot \overline{\boldsymbol{\Gamma}}_{T}\right\}
$$

Le dernier terme, indépendant de $\mathbf{p}^{\prime}$, ne contribue pas à $K_{\text {coll }}$; le premier conduit à un résultat analogue à celui obtenu pour le terme en $\breve{\Lambda}$.

\subsection{REPONSE LINEAIRE}

Dans ce paragraphe nous utiliserons la structure générale du terme source écrit précédemment pour calculer formellement la modification de la pression et le courant de chaleur résultant de la présence de gradients de la vitesse et de la température. En un point donné de l'espace, ces grandeurs s'expriment le plus simplement dans le référentiel où la vitesse $\mathbf{V}$ du gaz est nulle: nous nous y placerons dans toute la suite. A la limite (hydrodynamique) où nous nous sommes placés, les paramètres $N_{L}^{(0)}$ et $T^{(0)}$ utilisés dans la définition $(63)$ de $f^{(0)}$ ne diffèrent de la densité libre $N_{L}$ et de la température $T$ qu'à l'ordre un en gradients, si bien que nous pourrons les confondre dans la solution de l'équation cinétique linéarisée (88). Posant $\beta=1 / k_{B} T$, cette solution peut être décomposée sous la forme:

$$
\begin{aligned}
f^{(1)}(\mathbf{p}) & =f^{(0)}(\mathbf{p}) \varphi_{\nabla \cdot \mathbf{V}}(\mathbf{p}) \nabla \cdot \mathbf{V} \\
& +f^{(0)}(\mathbf{p}) \varphi_{\breve{\Lambda}}(\mathbf{p}) \frac{\beta}{2 m} \breve{\Lambda}:(\mathbf{p} \otimes \mathbf{p}) \\
& +f^{(0)}(\mathbf{p}) \varphi_{T}(\mathbf{p}) \sqrt{\frac{\beta}{2 m}} \mathbf{p} \cdot \frac{\nabla T}{T}
\end{aligned}
$$

Nous avons, de façon classique [4],[9], utilisé pour cela les symétries particulières du terme source et la linéarité de l'intégrale de collision linéarisée $I_{B}^{l}$ (terme d'amortissement). Chacune des fonctions $\varphi_{\nabla \cdot \mathbf{V}}, \varphi_{\breve{\Lambda}}$ et $\varphi_{T}$ ne dépend que du module de $\mathbf{p}$, et peut être estimée par les méthodes usuelles; nous le ferons de façon élémentaire au $§ 5.3$.

Calculons maintenant, dans le cadre que nous avons développé, la pression et le courant de chaleur.

\subsubsection{MODIFICATIONS VISQUEUSES DU TENSEUR DE PRESSION}


L'écart à la pression d'équilibre provoqué par un gradient de la vitesse est obtenu grâce à une linéarisation de la formule (11):

$$
\overline{\mathbf{P}}-P^{(0)}=\frac{1}{m} \int d^{3} p \mathbf{p} \otimes \mathbf{p} g^{(1)}(\mathbf{p})+\overline{\mathbf{Q}}_{l i n}^{(F)}+\overline{\mathbf{Q}}_{l i n}^{(k)}+\overline{\mathbf{Q}}_{l i n}^{(\Xi)}
$$

Les tenseurs linéarisés $\overline{\mathbf{Q}}_{\text {lin }}^{(i)}$ sont donnés par les formules (12), (13) et (14) où l'on fait à chaque fois les substitutions:

$$
\begin{aligned}
f(\mathbf{p}) f(\mathbf{p}-\mathbf{q}) & \rightarrow f^{(0)}(\mathbf{p}) f^{(1)}(\mathbf{p}-\mathbf{q})+f^{(0)}(\mathbf{p}-\mathbf{q}) f^{(1)}(\mathbf{p}) \\
f\left(\mathbf{1}^{\prime}\right) f\left(\mathbf{2}^{\prime}\right) & \rightarrow f^{(0)}\left(\mathbf{1}^{\prime}\right) f^{(1)}\left(\mathbf{2}^{\prime}\right)+f^{(0)}\left(\mathbf{2}^{\prime}\right) f^{(1)}\left(\mathbf{1}^{\prime}\right)
\end{aligned}
$$

Examinons d'abord le cas d'un mouvement de compression localement isotrope où $\breve{\Lambda}=0$. La fonction $g^{(1)}$ étant, comme $f^{(1)}$, scalaire en $\mathbf{p}$, l'intégrale au second membre de (119) est également scalaire, et il en est de même des trois corrections $\overline{\mathbf{Q}}_{l i n}^{(i)}$. Les contraintes (89) sur $g^{(1)}$ montrent que l'intégrale est nulle, et la modification de la pression se réduit alors à:

$$
P-P^{(0)}=-\frac{1}{4 m} \nabla \cdot \mathbf{V} \int d^{3} p \int d^{3} q q^{2} \Xi(q) f^{(0)}(\mathbf{p}) f^{(0)}(\mathbf{p}-\mathbf{q})\left[\varphi_{\nabla \cdot \mathbf{V}}(\mathbf{p})+\varphi_{\nabla \cdot \mathbf{V}}(\mathbf{p}-\mathbf{q})\right]
$$

Nous avons remarqué au $\S 5.1 .2$ que le terme source en $\nabla \cdot \mathbf{V}$ est une correction du premier ordre en densité: il en est de même pour $\varphi_{\nabla \cdot \mathbf{V}}$; la correction à la pression (121) est donc du second ordre en densité, et le coefficient de seconde viscosité qui s'en déduit directement est donc a priori au-delà de la validité des calculs. Rappelons en effet que l'équation cinétique établie dans l'article $\mathrm{B}$ est limitée à la première correction du viriel. Signalons ici toutefois que notre modèle, sous réserve d'un développement suffisant $\left.{ }^{*}\right)$, serait adapté au calcul à l'ordre non nul le plus bas (donc quadratique en densité) de la seconde viscosité puisque la modification de l'amortissement par les collisions à trois corps n'intervient qu'à l'ordre suivant.

La viscosité de cisaillement sera calculée à partir de l'expression à l'ordre un en densité de $\overline{\mathbf{P}}: \breve{\Lambda}$ tirée de (119), c'est à dire avec $g^{(1)}$ à l'ordre un dans l'intégrale, et $f^{(1)}$ à l'ordre zéro dans les corrections $\overline{\mathbf{Q}}_{\text {lin }}^{(i)}$. Utilisant le développement en densité $(87)$ pour $g^{(1)}$, on peut écrire:

$$
\begin{aligned}
\overline{\mathbf{P}}: \breve{\Lambda} & =\frac{\beta}{2 m^{2}} \int d^{3} p[(\mathbf{p} \otimes \mathbf{p}): \breve{\Lambda}]^{2} \varphi_{\breve{\Lambda}}(\mathbf{p}) f^{(0)}(\mathbf{p}) \\
& +\frac{\beta}{2 m^{2}} \int d^{3} p \int d^{3} q \Xi(q)(\mathbf{p} \otimes \mathbf{p}): \breve{\Lambda} \times \\
& \quad \times\left[\varphi_{\breve{\Lambda}}(\mathbf{p}) \mathbf{p} \otimes \mathbf{p}+\varphi_{\breve{\Lambda}}(\mathbf{p}-\mathbf{q})(\mathbf{p}-\mathbf{q}) \otimes(\mathbf{p}-\mathbf{q})\right]: \breve{\Lambda} f^{(0)}(\mathbf{p}) f^{(0)}(\mathbf{p}-\mathbf{q}) \\
& +\overline{\mathbf{Q}}_{l i n}^{(F)}: \breve{\Lambda}+\overline{\mathbf{Q}}_{l i n}^{(k)}: \breve{\Lambda}+\overline{\mathbf{Q}}_{l i n}^{(\Xi)}: \breve{\Lambda}
\end{aligned}
$$

\subsubsection{COURANT DE CHALEUR}

Supposant toujours la vitesse du fluide nulle, nous obtenons le courant de chaleur $\mathbf{J}_{T}$ par la linéarisation de la formule (15):

$$
\mathbf{J}_{T}=\int d^{3} p \frac{p^{2}}{2 m^{2}} \mathbf{p} g^{(1)}(\mathbf{p})+\mathbf{J}_{l i n}^{(F)}+\mathbf{J}_{l i n}^{(k)}+\mathbf{J}_{l i n}^{(\Xi)}
$$

$\left(^{*}\right)$ Dans le calcul du $\S 1$ de l'article B (équation 13 par exemple), il faut aller un ordre plus loin dans le développement en gradients, afin d'obtenir les corrections supplémentaires au tenseur des contraintes intervenant elles aussi à l'ordre deux en densité. 
Les courants linéarisés $\mathbf{J}_{\text {lin }}^{(i)}$ sont donnés par les formules (16), (17) et (18), où l'on fait à chaque fois la substitution (120). De la même façon que précédemment, il faudra utiliser $g^{(1)}$ à l'ordre un en densité, mais $f^{(1)}$ à l'ordre zéro dans les courants $\mathbf{J}_{l i n}^{(i)}$. Reportons dans (123) la forme (118) de $f^{(1)}$, afin de calculer:

$$
\begin{aligned}
& \mathbf{J}_{T} \cdot \frac{\nabla T}{T}=\frac{1}{2 m^{2}} \sqrt{\frac{\beta}{2 m}} \int d^{3} p p^{2}\left(\mathbf{p} \cdot \frac{\nabla T}{T}\right)^{2} \varphi_{T}(\mathbf{p}) f^{(0)}(\mathbf{p}) \\
& +\frac{1}{2 m^{2}} \sqrt{\frac{\beta}{2 m}} \int d^{3} p \int d^{3} q \Xi(q) p^{2} \mathbf{p} \cdot \frac{\nabla T}{T} \times \\
& \quad \times\left[\varphi_{T}(\mathbf{p}) \mathbf{p} \cdot \frac{\nabla T}{T}+\varphi_{T}(\mathbf{p}-\mathbf{q})(\mathbf{p}-\mathbf{q}) \cdot \frac{\nabla T}{T}\right] f^{(0)}(\mathbf{p}) f^{(0)}(\mathbf{p}-\mathbf{q}) \\
& +\mathbf{J}_{l i n}^{(F)} \cdot \frac{\nabla T}{T}+\mathbf{J}_{l i n}^{(k)} \cdot \frac{\nabla T}{T}+\mathbf{J}_{l i n}^{(\Xi)} \cdot \frac{\nabla T}{T}
\end{aligned}
$$

\subsection{AMORTISSEMENT}

Dans l'écriture (118) de la solution de l'équation cinétique linéarisée (88), nous avons introduit les fonctions $\varphi_{\breve{\Lambda}}$ et $\varphi_{T}$ du module de $\mathbf{p}$. Leur calcul est nécessaire afin de déduire des formules (122) et (124) les expressions de la viscosité et de la conductivité thermique. Nous chercherons les solutions $\varphi_{\breve{\Lambda}}$ et $\varphi_{T}$ sous forme d'un développement limité en puissances de $\mathbf{p}^{2}$ :

$$
\begin{aligned}
& \varphi_{\breve{\Lambda}}(\mathbf{p})=a \\
& \varphi_{T}(\mathbf{p})=b+b^{\prime} \frac{\beta}{2 m} \mathbf{p}^{2}
\end{aligned}
$$

Les coefficients $a$ et $b^{\prime}$ seront déterminés en égalant les plus bas moments non nuls des deux membres de l'équation cinétique; notant $S_{\breve{\Lambda}}$ et $S_{T}$ les termes source calculés aux $\S \S 5.1 .2$ et 5.1.3, ces conditions s'écrivent:

$$
\begin{aligned}
& \int d^{3} p \frac{\beta}{2 m}(\mathbf{p} \otimes \mathbf{p}): \breve{\Lambda}\left\{I_{B}^{l}\left[f^{(0)}, f^{(1)}\right](\mathbf{p})-S_{\breve{\Lambda}}(\mathbf{p})\right\}=0 \\
& \int d^{3} p\left(\frac{\beta}{2 m}\right)^{3 / 2} p^{2} \mathbf{p} \cdot \frac{\nabla T}{T}\left\{I_{B}^{l}\left[f^{(0)}, f^{(1)}\right](\mathbf{p})-S_{T}(\mathbf{p})\right\}=0
\end{aligned}
$$

Dans ces équations seuls contribuent les termes en $a$ et $b^{\prime}$; les contraintes (89) sont automatiquement vérifiées pour la partie en $\varphi_{\breve{\Lambda}}$ de $g^{(1)}$, et $b$ sera déterminé par la seconde d'entre elles pour la partie en $\varphi_{T}$. Nous employons en fait une généralisation de la méthode habituelle de développement en polynômes de Sonine (qui vérifient automatiquement les contraintes semblables à (89)), limitée au degré significatif le plus bas. Elle pourrait, au prix de calculs plus lourds, donner une meilleure approximation aux coefficients de transport en prenant pour les fonctions $\varphi_{\breve{\Lambda}}$ et $\varphi_{T}$ des polynomes en $\mathbf{p}^{2}$ de degré plus élevé.

Il est bien entendu possible de calculer directement les intégrales des termes en $I_{B}^{l}$. Nous éviterons cependant de le faire ici en remarquant qu'elles ne contiennent aucune correction en

densité, et donnent des résultats proportionnels à $a$ et $b^{\prime}$, que nous noterons $a I_{\breve{\Lambda}}$ et $b^{\prime} I_{T}$. Les facteurs $I_{\breve{\Lambda}}$ et $I_{T}$ pourraient être déterminés en identifiant la solution du calcul à l'ordre zéro en densité avec les valeurs à l'ordre zéro de la viscosité et de la conductivité, données par exemple dans [4]. Nous n'en aurons toutefois pas besoin dans la suite.

\subsection{MODIFICATION DES COEFFICIENTS DE TRANSPORT}


Nous allons maintenant calculer, à partir des formules (122) et (124), le rapport de la viscosité $\eta$ et de la conductivité $\kappa$ à leurs valeurs respectives $\eta_{0}$ et $\kappa_{0}$ à l'ordre zéro en densité. Nous noterons $a_{0}, b_{0}, b_{0}^{\prime}, S_{T 0}(\mathbf{p})$ et $S_{\breve{\Lambda} 0}(\mathbf{p})$ les valeurs à l'ordre zéro en densité des grandeurs correspondantes dans les équations (125) à (128), $a_{1}, b_{1} \ldots$ leurs corrections du premier ordre en densité.

\subsubsection{VISCOSITE}

Développée en densité, l'équation (122) peut être écrite:

$$
\begin{aligned}
\frac{\eta}{\eta_{0}}= & 1+\frac{a_{1}}{a_{0}} \\
+ & \left\{\int d^{3} p \int d^{3} q \Xi(q) \mathbf{p} \otimes \mathbf{p}: \breve{\Lambda}[\mathbf{p} \otimes \mathbf{p}+(\mathbf{p}-\mathbf{q}) \otimes(\mathbf{p}-\mathbf{q})]: \breve{\Lambda} f^{(0)}(\mathbf{p}) f^{(0)}(\mathbf{p}-\mathbf{q})\right. \\
& \left.+\frac{2 m^{2}}{\beta a_{0}}\left[\mathbf{Q}_{l i n}^{(F)}: \breve{\Lambda}+\mathbf{Q}_{l i n}^{(k)}: \breve{\Lambda}+\mathbf{Q}_{l i n}^{(\Xi)}: \breve{\Lambda}\right]\right\} \times\left[\int d^{3} p(\mathbf{p} \otimes \mathbf{p}: \breve{\Lambda})^{2} f^{(0)}(\mathbf{p})\right]^{-1}
\end{aligned}
$$

où le rapport $a_{1} / a_{0}$ est donné à partir de (127) par:

$$
\frac{a_{1}}{a_{0}}=\int d^{3} p \mathbf{p} \otimes \mathbf{p}: \breve{\Lambda} S_{\breve{\Lambda} 1}(\mathbf{p}) \quad\left[\int d^{3} p \mathbf{p} \otimes \mathbf{p}: \breve{\Lambda} S_{\breve{\Lambda} 0}(\mathbf{p})\right]^{-1}
$$

Pour calculer explicitement les différentes intégrales, on peut supposer que $\breve{\Lambda}$ n'a que deux composantes symétriques non nulles. On obtient alors, pour les trois termes de l'expression (129):

$$
\begin{aligned}
\frac{\eta}{\eta_{0}} & =1+\frac{1}{2} N \Sigma_{0}+\frac{2}{5} \int d^{3} q \Phi_{2 m}(\mathbf{q})\left(\frac{\beta q^{2}}{4 m}\right)^{2}\left[-2 \frac{\Delta_{F}}{q}+\int d^{2} \hat{q}^{\prime}\left(\xi_{k}-\Delta_{k} \cos \theta\right)\left(1-\cos ^{2} \theta\right)\right] \\
& +\frac{1}{2} N \Sigma_{0}+\frac{2}{15} N \Sigma_{2} \\
& -\frac{2}{15} N \Sigma_{2}-\frac{2}{5} \int d^{3} q \Phi_{2 m}(\mathbf{q})\left(\frac{\beta q^{2}}{4 m}\right)^{2}\left[2 \frac{\Delta_{F}}{q}+\int d^{2} \hat{q}^{\prime} \Delta_{k} \cos \theta\left(1-\cos ^{2} \theta\right)\right]
\end{aligned}
$$

Ces trois contributions peuvent être respectivement interprétées comme venant de la modification du terme source par rapport à celui de l'équation de Boltzmann, des corrections en densité dans la fonction de distribution (différence entre $g^{(1)}$ et $f^{(1)}$ ), et des termes complémentaires dans la définition de la pression (transport par les collisions). Ils peuvent être regroupés sous la forme:

$$
\frac{\eta}{\eta_{0}}=1+N \Sigma_{0}+\frac{2}{5} \int d^{3} q \Phi_{2 m}(\mathbf{q})\left(\frac{\beta q^{2}}{4 m}\right)^{2}\left[-4 \frac{\Delta_{F}}{q}+\int d^{2} \hat{q}^{\prime}\left(\xi_{k}-2 \Delta_{k} \cos \theta\right)\left(1-\cos ^{2} \theta\right)\right]
$$

\subsubsection{CONDUCTIVITE THERMIQUE}

Développée en densité, l'équation (124) peut être écrite:

$$
\begin{aligned}
\frac{\kappa}{\kappa_{0}}=1+\left[b_{0} I_{b}+b_{0}^{\prime} I_{b^{\prime}}\right]^{-1} \times\left\{b_{1} I_{b}+b_{1}^{\prime} I_{b^{\prime}}+\right. \\
+\int d^{3} p \int d^{3} q \Xi(q) p^{2} \mathbf{p} \cdot \frac{\nabla T}{T} f^{(0)}(\mathbf{p}) f^{(0)}(\mathbf{p}-\mathbf{q})\left[b_{0}(2 \mathbf{p}-\mathbf{q}) \cdot \frac{\nabla T}{T}\right. \\
\left.+b_{0}^{\prime} \frac{\beta}{2 m}\left[p^{2} \mathbf{p} \cdot \frac{\nabla T}{T}+(\mathbf{p}-\mathbf{q})^{2}(\mathbf{p}-\mathbf{q}) \cdot \frac{\nabla T}{T}\right]\right] \\
\left.+2 m^{2} \sqrt{\frac{2 m}{\beta}}\left[\mathbf{J}_{\text {lin }}^{(F)} \cdot \frac{\nabla T}{T}+\mathbf{J}_{\text {lin }}^{(k)} \cdot \frac{\nabla T}{T}+\mathbf{J}_{\text {lin }}^{(\Xi)} \cdot \frac{\nabla T}{T}\right]\right\}
\end{aligned}
$$


où l'on a posé:

$$
\begin{aligned}
I_{b} & =\int d^{3} p p^{2}\left(\mathbf{p} \cdot \frac{\nabla T}{T}\right)^{2} f^{(0)}(\mathbf{p}) \\
I_{b^{\prime}} & =\int d^{3} p p^{2}\left(\mathbf{p} \cdot \frac{\nabla T}{T}\right)^{2} \frac{\beta p^{2}}{2 m} f^{(0)}(\mathbf{p})=\frac{7}{2} I_{b}
\end{aligned}
$$

La seconde des contraintes (89) donne quant à elle:

$$
\begin{aligned}
& b_{0}=-\frac{5}{2} b_{0}^{\prime} \\
& b_{1}=\frac{5}{2} b_{0}^{\prime}\left[N \Sigma_{0} / 2-N \Sigma_{1} / 3\right]-\frac{5}{2} b_{1}^{\prime}
\end{aligned}
$$

L'équation (133) peut donc s'écrire, en utilisant (134) et (135):

$$
\begin{aligned}
\frac{\kappa}{\kappa_{0}}= & 1+\frac{5}{4} N \Sigma_{0}-\frac{5}{6} N \Sigma_{1}+\frac{b_{1}^{\prime}}{b_{0}^{\prime}} \\
+ & \frac{1}{I_{b}} \int d^{3} p \int d^{3} q \Xi(q) p^{2} \mathbf{p} \cdot \frac{\nabla T}{T} f^{(0)}(\mathbf{p}) f^{(0)}(\mathbf{p}-\mathbf{q})\left[-5\left(\mathbf{p}-\frac{\mathbf{q}}{2}\right) \cdot \frac{\nabla T}{T}\right. \\
& \left.\quad+\frac{\beta}{2 m}\left[p^{2} \mathbf{p} \cdot \frac{\nabla T}{T}+(\mathbf{p}-\mathbf{q})^{2}(\mathbf{p}-\mathbf{q}) \cdot \frac{\nabla T}{T}\right]\right] \\
+ & 2 m^{2} \sqrt{\frac{2 m}{\beta}} \frac{1}{b_{0}^{\prime} I_{b}}\left[\mathbf{J}_{l i n}^{(F)} \cdot \frac{\nabla T}{T}+\mathbf{J}_{l i n}^{(k)} \cdot \frac{\nabla T}{T}+\mathbf{J}_{l i n}^{(\Xi)} \cdot \frac{\nabla T}{T}\right]
\end{aligned}
$$

Enfin, l'équation (128) entraîne:

$$
\frac{b_{1}^{\prime}}{b_{0}^{\prime}}=\int d^{3} p p^{2} \mathbf{p} \cdot \frac{\nabla T}{T} S_{T 1}(\mathbf{p})\left[\int d^{3} p p^{2} \mathbf{p} \cdot \frac{\nabla T}{T} S_{T 0}(\mathbf{p})\right]^{-1}
$$

On obtient alors pour les trois termes de l'expression (136):

$$
\begin{aligned}
\frac{\kappa}{\kappa_{0}}= & 1+\left(\frac{5}{4} N \Sigma_{0}-\frac{5}{6} N \Sigma_{1}\right)+\frac{7}{8} N \Sigma_{0}-N \Sigma_{1}+\frac{1}{6} N \Sigma_{2} \\
& \quad+\frac{4}{15} \int d^{3} q \Phi_{2 m}(\mathbf{q})\left(\frac{\beta q^{2}}{4 m}\right)^{2}\left[-2 \frac{\Delta_{F}}{q}+\int d^{2} \hat{q}^{\prime}\left(\xi_{k}-\Delta_{k} \cos \theta\right)\left(1-\cos ^{2} \theta\right)\right] \\
- & \frac{3}{8} N \Sigma_{0}-\frac{1}{3} N \Sigma_{1}+\frac{11}{30} N \Sigma_{2} \\
+ & \frac{1}{6} N \Sigma_{1}-\frac{1}{5} N \Sigma_{2}-\frac{4}{15} \int d^{3} q \Phi_{2 m}(\mathbf{q})\left(\frac{\beta q^{2}}{4 m}\right)^{2}\left[2 \frac{\Delta_{F}}{q}+\int d^{2} \hat{q}^{\prime} \Delta_{k} \cos \theta\left(1-\cos ^{2} \theta\right)\right]
\end{aligned}
$$

L'origine de chacun de ces termes peut être interprétée comme dans le cas de la formule (131) qui donne la modification de la viscosité dûe aux effets de densité. Ils peuvent finalement être regroupés sous la forme:

$$
\begin{aligned}
\frac{\kappa}{\kappa_{0}}= & 1+\frac{7}{4} N \Sigma_{0}-\frac{4}{3} N \Sigma_{1}+\frac{1}{3} N \Sigma_{2} \\
& +\frac{4}{15} \int d^{3} q \Phi_{2 m}(\mathbf{q})\left(\frac{\beta q^{2}}{4 m}\right)^{2}\left[-4 \frac{\Delta_{F}}{q}+\int d^{2} \hat{q}^{\prime}\left(\xi_{k}-2 \Delta_{k} \cos \theta\right)\left(1-\cos ^{2} \theta\right)\right]
\end{aligned}
$$


Rappelons enfin ici les approximations faites pour obtenir les expressions explicites de la viscosité et de la conductivité thermique. La première a consisté à se limiter aux plus basses corrections de densité aux coefficients de transport. Comme nous l'avons remarqué au $§ 5.2 .1$ à propos de la seconde viscosité, il serait possible d'obtenir l'ordre suivant (corrections quadratiques en densité) sous réserve de développer à l'ordre deux en gradients l'équation cinétique. La seconde approximation que nous avons faite a été de résoudre explicitement l'équation cinétique linéarisée dans un espace de fonctions restreint. Il s'agit là d'une méthode classique qui donne en général une bonne estimation des coefficients de transport [4]. Aucune de ces approximations n'est cependant indispensable, et il est en principe possible de les améliorer.

Par contre, il convient d'insister sur le fait que notre théorie ne prend en compte que les collisions binaires entre atomes, et que de ce fait on ne peut en général espérer décrire complètement toutes les corrections en densité aux propriétés de transport. Ainsi par exemple le terme d'amortissement écrit au $\S 5.3$ doit-il contenir une correction due aux collisions à trois corps, contenant un facteur supplémentaire en densité, et dont l'effet sur les coefficients de transport est du même ordre en densité que les corrections que nous avons calculées. Ce n'est que dans le cas où les effets à trois corps seraient faibles (limite classique de sphères dures, comme dans le modèle d'Enskog par exemple), ou bien les effets à deux corps importants (quasi-résonances du potentiel atomique, entraînant d'importantes "sections efficaces" pour les termes $\xi_{F}, \xi_{k}, \Delta_{F}$ ou $\Delta_{k}$ ) que nous pourrons espérer prévoir quantitativement les corrections du viriel à la viscosité ou à la conductivité d'un gaz modérément dense.

\section{CONCLUSION}

L'équation cinétique obtenue dans l'article B remplit donc son rôle, puisqu'elle permet de retrouver les résultats déjà connus sur les seconds coefficients du viriel à la pression ou la chaleur spécifique; de plus, elle permet de les généraliser à des situations hors d'équilibre; enfin, elle fournit également des résultats nouveaux, tels que les expressions exactes de certaines corrections du viriel aux coefficients de transport (à l'exclusion des effets à trois corps). Le prix à payer, comme on pouvait s'y attendre, est l'introduction de termes nouveaux dans les équations qui ne vont pas dans le sens de la simplification des calculs.

D'un point de vue très général, il nous paraît satisfaisant d'avoir un formalisme qui permette l'évaluation rigoureuse, par une méthode systématique, de tous les effets exactement calculables (nous entendons par là tous les effets à deux corps), que le système étudié soit ou non à l'équilibre. Ces effets incluent en particulier la présence dans l'équation cinétique de termes du type "champ moléculaire" similaires à ceux d'une théorie de Hartree Fock ou encore à ceux de l'équation de Landau. Notre démarche à été de partir de l'idée que tout terme de ce type, ou du moins son développement au premier ordre en densité, doit avoir une contrepartie dans les résultats d'une étude détaillée d'une collision binaire; le calcul montre que cette approche est effectivement correcte. S'il existe des différences avec les termes de champ moléculaire habituels, on pouvait s'y attendre pour des raisons physiques qui ont été discutées dans la conclusion de l'article B (effets de recul se produisant lorsque les forces d'interaction sont à courte portée).

Signalons enfin qu'on peut songer à utiliser cette équation cinétique de façon phénoménologique pour des systèmes plus denses que ceux pour lesquels nous en avons établi la validité. Une telle démarche n'est pas possible avec l'équation de Boltzmann où les effets de champ moléculaire sont totalement ignorés, mais elle devient envisageable dès qu'une expression, même approchée, de ces effets est disponible. 
Appendice C1:

\section{GRANDEURS HYDRODYNAMIQUES ET LOIS DE CONSERVATION}

Nous présentons dans cet appendice le calcul direct du tenseur des contraintes et du courant d'énergie à partir de l'équation cinétique (6) écrite pour la fonction de distribution totale $g(\mathbf{r}, \mathbf{p}, t)$. Ces grandeurs sont définies afin de vérifier les lois de conservation locale pour les densités $N$, $\mathbf{J}$ et $U$ définies à partir de $g$ par les formules (8), (9) et (10).

\section{C1.1. CONSERVATION DE LA DENSITE DE PARTICULES}

Intégrant sur $\mathbf{p}$ l'équation cinétique (6), nous obtenons pour le premier membre:

$$
\frac{\partial}{\partial t} \int d^{3} p g(\mathbf{p})+\int d^{3} p \frac{\mathbf{p}}{m} \cdot \nabla g(\mathbf{p})=\frac{\partial N}{\partial t}+\nabla \cdot \mathbf{J}
$$

Le second membre contient trois termes:

(i) l'intégrale de $I_{B}$ donne zéro, comme il est bien connu;

(ii) le calcul de l'intégrale de $K_{\text {coll }}$ est le même que dans le $\S 3.1$ de l'article B et conduit au résultat:

$$
\int d^{3} p K_{\text {coll }}[f(\mathbf{p})]=-\frac{1}{2 m} \int d^{3} p \int d^{3} q \Xi(q) \mathbf{q} \cdot[f(\mathbf{p}) \nabla f(\mathbf{p}-\mathbf{q})-f(\mathbf{p}-\mathbf{q}) \nabla f(\mathbf{p})](C 1-2)
$$

(iii) l'intégrale sur $\mathbf{p}$ de $R_{\Xi}$ vaut:

$$
\int d^{3} p R_{\Xi}[f(\mathbf{p})]=\frac{1}{m} \int d^{3} p \int d^{3} q \Xi(q) \mathbf{q} \cdot[f(\mathbf{p}) \nabla f(\mathbf{p}-\mathbf{q})]
$$

Au total, le second membre vaut donc:

$$
\frac{1}{2 m} \int d^{3} p \int d^{3} q \Xi(q) \mathbf{q} \cdot \nabla[f(\mathbf{p}) f(\mathbf{p}-\mathbf{q})]
$$

Prenant comme variables $\mathbf{q}$ et $\mathbf{P}=2 \mathbf{p}+\mathbf{q}$, il apparait que l'intégrant est impair en $\mathbf{q}$, ce qui montre que l'intégrale est nulle. En définitive on a bien:

$$
\frac{\partial N(\mathbf{r}, t)}{\partial t}+\nabla \cdot \mathbf{J}(\mathbf{r}, t)=0
$$

\section{C1.2. CONSERVATION DE L'IMPULSION; TENSEUR DE PRESSION}

Préoccupons nous maintenant de la conservation locale de l'impulsion, qui nous conduira à l'expression du tenseur des contraintes $\overline{\mathbf{Q}}$. Multipliant l'équation cinétique par $\mathbf{p}$ et sommant sur p, il apparait au premier membre:

$$
\frac{\partial}{\partial t} \int d^{3} p \mathbf{p} g(\mathbf{p})+\int d^{3} p \mathbf{p}\left(\frac{\mathbf{p}}{m} \cdot \nabla g(\mathbf{p})\right)=m \frac{\partial \mathbf{J}}{\partial t}+\nabla \cdot \int d^{3} p \frac{\mathbf{p} \otimes \mathbf{p}}{m} g(\mathbf{p}) \quad(C 1-6)
$$

Pour le calcul du second membre, il est à nouveau commode d'effectuer le changement de variable $\mathbf{P}=2 \mathbf{p}+\mathbf{q}$. Par un calcul identique à celui du $\S 4$ de l'article $\mathrm{B}$, on trouve que la contribution de l'intégrale du terme en $I_{B}$ est nulle, et que celle du terme en $K_{\text {coll }}$ vaut:

$$
\int d^{3} p \mathbf{p} K_{\text {coll }}[f(\mathbf{p})]=-\nabla \cdot\left[\overline{\mathbf{Q}}^{(F)}+\overline{\mathbf{Q}}^{(k)}\right]
$$


où les tenseurs $\overline{\mathbf{Q}}^{(F)}$ et $\overline{\mathbf{Q}}^{(k)}$ sont donnés par les formules (12) et (13). Quant au terme en $R_{\Xi}$, son intégrale donne:

$$
\frac{1}{16 m} \int d^{3} P \int d^{3} q f\left(\frac{\mathbf{P}}{2}+\frac{\mathbf{q}}{2}\right) \Xi(q)(\mathbf{P}+\mathbf{q}) \mathbf{q} \cdot \nabla f\left(\frac{\mathbf{P}}{2}-\frac{\mathbf{q}}{2}\right) \quad(C 1-8)
$$

ce qui vaut encore:

$\frac{1}{32 m} \int d^{3} P \int d^{3} q \Xi(q) \mathbf{P} \mathbf{q} \cdot \nabla\left[f\left(\frac{\mathbf{P}}{2}+\frac{\mathbf{q}}{2}\right) f\left(\frac{\mathbf{P}}{2}-\frac{\mathbf{q}}{2}\right)\right]+\frac{1}{4 m} \int d^{3} p \int d^{3} q \Xi(q) \mathbf{q} \otimes \mathbf{q} \nabla[f(\mathbf{p}) f(\mathbf{p}-\mathbf{q})]$

La première intégrale est impaire en $\mathbf{P}$, donc nulle; la seconde est l'opposée de la divergence du tenseur $\overline{\mathbf{Q}}^{(\Xi)}$ défini par la formule (14). En définitive, on obtient:

$$
\frac{\partial \mathbf{J}(\mathbf{r}, t)}{\partial t}+\nabla \cdot \overline{\mathbf{Q}}(\mathbf{r}, t)=0
$$

où $\overline{\mathbf{Q}}$ est le tenseur des contraintes défini en (11).

Définissons la vitesse moyenne locale par:

$$
\mathbf{J}(\mathbf{r}, t)=N(\mathbf{r}, t) \mathbf{V}(\mathbf{r}, t)
$$

Le tenseur de pression $\overline{\mathbf{P}}$ étant défini localement comme étant égal au tenseur des contraintes dans le référentiel où le courant est nul, on vérifie aisément que:

$$
\overline{\mathbf{P}}=\overline{\mathbf{Q}}-m N \mathbf{V} \otimes \mathbf{V}
$$

(On peut remarquer que $\overline{\mathbf{Q}}^{(F)}, \overline{\mathbf{Q}}^{(k)}$ et $\overline{\mathbf{Q}}^{(\Xi)}$ sont invariants par changement de repère galiléen, car seule y compte l'impulsion relative des particules).

Utilisant (C1-10) et (C1-12), il est alors classique de montrer que:

$$
m N\left(\frac{\partial}{\partial t}+\mathbf{V} \cdot \nabla\right) \mathbf{V}+\nabla \cdot \overline{\mathbf{P}}=0
$$

\section{C1.3.CONSERVATION DE L'ENERGIE; COURANT DE CHALEUR}

Multiplions l'équation cinétique par $p^{2} / 2 m$ et intégrons sur $\mathbf{p}$; le premier membre donne:

$$
\frac{\partial}{\partial t} \int d^{3} p \frac{p^{2}}{2 m} g(\mathbf{p})+\int d^{3} p \frac{p^{2}}{2 m^{2}} \mathbf{p} \cdot \nabla g(\mathbf{p})=\frac{\partial U}{\partial t}+\nabla \cdot \int d^{3} p \frac{p^{2}}{2 m^{2}} \mathbf{p} g(\mathbf{p}) \quad(C 1-14)
$$

Le calcul du second membre est effectué avec les variables d'intégration $\mathbf{P}$ et $\mathbf{q}$, faisant ainsi apparaître trois termes.

(i) terme en $\mathbf{p} \cdot \mathbf{q}$ : le même calcul qu'au $\S 4.1$ de l'article B montre que le terme en $I_{B}$ ne contribue pas, et que le terme en $K_{\text {coll }}$ donne:

$$
\int d^{3} p \frac{p^{2}}{2 m} K_{\text {coll }}[f(\mathbf{p})]=-\nabla \cdot\left(\mathbf{J}_{U}^{(F)}+\mathbf{J}_{U}^{(k)}\right)
$$

où les courants $\mathbf{J}_{U}^{(F)}$ et $\mathbf{J}_{U}^{(k)}$ sont donnés par les formules (16) et (17). L'intégrale du terme en $R_{\Xi}$ donne quant à elle:

$$
\frac{1}{32 m^{2}} \int d^{3} P \int d^{3} q \Xi(q) \mathbf{P} \cdot \mathbf{q} f\left(\frac{\mathbf{P}+\mathbf{q}}{2}\right) \mathbf{q} \cdot \nabla f\left(\frac{\mathbf{P}-\mathbf{q}}{2}\right)
$$


qui vaut encore:

$$
\frac{1}{8 m^{2}} \int d^{3} p \int d^{3} q \Xi(q)(2 \mathbf{p}-\mathbf{q}) \cdot \mathbf{q} \mathbf{q} \cdot \nabla[f(\mathbf{p}) f(\mathbf{p}-\mathbf{q})]=-\nabla \cdot \mathbf{J}_{U}^{(\Xi)}
$$

où $\mathbf{J}_{U}^{(\Xi)}$ est le courant défini par la formule (18).

(ii) termes en $P^{2}$ et $q^{2}$; le calcul est semblable à celui effectué pour la densité, et le regroupement des termes en $K_{\text {coll }}$ et $R_{\Xi}$ donne:

$$
\frac{1}{32 m^{2}} \int d^{3} P \int d^{3} q \Xi(q) \frac{P^{2}+q^{2}}{4} \mathbf{q} \cdot \nabla\left[f\left(\frac{\mathbf{P}+\mathbf{q}}{2}\right) f\left(\frac{\mathbf{P}-\mathbf{q}}{2}\right)\right]
$$

L'intégrant étant impair en $\mathbf{q}$, cette contribution est nulle, et il reste en définitive:

$$
\frac{\partial U}{\partial t}+\nabla \cdot \mathbf{J}_{U}=0
$$

où $\mathbf{J}_{U}$ est le courant total d'énergie défini par l'égalité (15).

Passant localement dans le référentiel où la vitesse moyenne est nulle, on obtient la densité d'énergie thermique $W$ et le courant de chaleur $\mathbf{J}_{T}$ donnés par:

$$
\begin{gathered}
W=U-\frac{1}{2} m N V^{2} \\
\mathbf{J}_{T}=\mathbf{J}_{U}-U \mathbf{V}-\overline{\mathbf{P}} \cdot \mathbf{V}
\end{gathered}
$$

Un calcul classique conduit alors à l'équation hydrodynamique:

$$
\frac{\partial W}{\partial t}+\nabla \cdot \mathbf{J}_{T}+\nabla \cdot(W \mathbf{V})+\overline{\mathbf{P}}: \bar{\Lambda}=0
$$

où $\bar{\Lambda}$ est le tenseur (symétrique) des gradients de la vitesse (formule (73) de l'article B).

Si nous définissons, comme dans l'article B, la température $T$ à partir des quantités "libres" $N_{L}$ et $W_{L}$ (obtenues en prenant $f$ et non $g$ comme fonction de distribution) par l'égalité:

$$
\frac{3}{2} N_{L} k_{B} T=W_{L}
$$

l'énergie totale peut s'écrire:

$$
W=\frac{3}{2} N k_{B} T\left[\frac{W N_{L}}{W_{L} N}\right]
$$

On peut alors mettre l'équation (C1-22) sous la forme:

$$
N\left(\frac{\partial}{\partial t}+\mathbf{V} \cdot \nabla\right)\left(\frac{3}{2} k_{B} T \frac{W N_{L}}{W_{L} N}\right)+\nabla \cdot \mathbf{J}_{T}+\overline{\mathbf{P}}: \bar{\Lambda}=0
$$




\section{Appendice $\mathrm{C} 2$ :}

\section{FORMULAIRE}

Nous présentons dans cet appendice quelques résultats concernant la famille de fonctions gaussiennes $\Phi_{a}$ introduite au $\S 2.2$ :

$$
\Phi_{a}(\mathbf{x})=N_{L}\left(\frac{\beta}{2 \pi a}\right)^{3 / 2} e^{-\beta x^{2} / 2 a}
$$

\section{C2.1 MOMENTS DES FONCTIONS $\Phi_{a}$ :}

Les différents moments de ces fonctions sont bien connus. Rappelons que le premier moment vaut:

$$
\int d^{3} x \Phi_{a}(\mathbf{x})=N_{L}
$$

et le second:

$$
\int d^{3} x \quad x^{2} \Phi_{a}(\mathbf{x})=\frac{3 a}{\beta} N_{L}
$$

De façon plus générale, pour tout entier naturel n, on a:

$$
\begin{aligned}
\int d^{3} x x^{2 n} \Phi_{a}(\mathbf{x}) & =(2 n+1)(2 n-1) \cdots\left(\frac{a}{\beta}\right)^{n} N_{L} \\
& =\frac{(2 n+1) !}{n !}\left(\frac{a}{2 \beta}\right)^{n} N_{L}
\end{aligned}
$$

\section{C2.2 MOMENTS GAUSSIENS DE $\Xi$ :}

Les calculs développés dans le présent article font fréquemment intervenir des intégrales du type:

$$
\int d^{3} q \Xi\left(\frac{q}{2 \hbar}\right) q^{2 n} \Phi_{2 m}(\mathbf{q})
$$

Nous avons choisi de définir une suite d'intégrales $\Sigma_{n}$ telles que:

$$
N_{L} \Sigma_{n}=\left(\frac{\beta}{4 m}\right)^{n} \int d^{3} q \Xi\left(\frac{q}{2 \hbar}\right) q^{2 n} \Phi_{2 m}(\mathbf{q})
$$

Afin de ne faire apparaître que des variables d'intégration sans dimension, nous introduisons:

$$
\gamma=\frac{q}{2} \sqrt{\frac{\beta}{m}}
$$

et réécrivons $(\mathrm{C} 2-6)$ sous la forme:

$$
\begin{aligned}
\Sigma_{n} & =\pi^{-3 / 2} \int d^{3} \gamma \gamma^{2 n} \Xi\left(\frac{\gamma}{\hbar} \sqrt{\frac{m}{\beta}}\right) e^{-\gamma^{2}} \\
& =\frac{4}{\sqrt{\pi}} \int_{0}^{\infty} d \gamma \gamma^{2 n+2} \Xi\left(\frac{\gamma}{\hbar} \sqrt{\frac{m}{\beta}}\right) e^{-\gamma^{2}}
\end{aligned}
$$

La formule générique pour les $\Sigma_{n}$ s'écrit:

$$
\Sigma_{n}=\beta^{3 / 2}(-\beta)^{n} \frac{\partial^{n}}{\partial \beta^{n}}\left[\beta^{-3 / 2} \Sigma_{0}\right]
$$


Elle conduit naturellement à la formule de récurrence simple suivante:

$$
\Sigma_{n}=-\beta \frac{\partial}{\partial \beta} \Sigma_{n-1}+\left(n+\frac{1}{2}\right) \Sigma_{n-1}
$$

ou encore:

$$
T \frac{\partial}{\partial T} \Sigma_{n}=\Sigma_{n-1}-\left(n+\frac{3}{2}\right) \Sigma_{n}
$$

Nous avons donc en particulier:

$$
\begin{aligned}
& T \frac{\partial}{\partial T} \Sigma_{0}=\Sigma_{1}-\frac{3}{2} \Sigma_{0} \\
& T \frac{\partial}{\partial T} \Sigma_{1}=\Sigma_{2}-\frac{5}{2} \Sigma_{1}
\end{aligned}
$$

et aussi:

$$
\begin{aligned}
& \Sigma_{1}=\frac{3}{2} \Sigma_{0}-\beta \frac{\partial}{\partial \beta} \Sigma_{0} \\
& \Sigma_{2}=3 \Sigma_{1}-\frac{3}{4} \Sigma_{0}+\beta^{2} \frac{\partial^{2}}{\partial \beta^{2}} \Sigma_{0}
\end{aligned}
$$

Enfin, rappelons que le second coefficient du viriel $B_{2}(T)$ est intimement lié à $\Sigma_{0}$, puisque (formule $(50))$ :

$$
B_{2}(T)=-\frac{1}{2} \Sigma_{0}
$$

Nous pouvons dans ces conditions réécrire (C2-9) sous la forme:

$$
\Sigma_{n}=-2 \beta^{3 / 2}(-\beta)^{n} \frac{\partial^{n}}{\partial \beta^{n}}\left[\beta^{-3 / 2} B_{2}(T)\right]
$$


Appendice C3:

\section{CALCUL DU TENSEUR DES CONTRAINTES ET DU COURANT DE CHALEUR POUR UN SYSTEME UNIFORME DANS L'ESPACE}

Nous présentons dans cet appendice le détail du calcul explicite du tenseur des contraintes $\overline{\mathbf{Q}}$ et du courant de chaleur $\mathbf{J}_{U}$ définis au $\S 1.1$, pour la fonction de distribution uniforme stationnaire (22). Nous insistons à nouveau sur l'extension possible des résultats obtenus à toute situation où la fonction $f$ n'est que localement gaussienne.

\section{C3.1 TENSEUR DES CONTRAINTES:}

D'après la définition (11), le tenseur des contraintes $\mathbf{Q}$ s'exprime comme la somme de quatre termes:

$$
\overline{\mathbf{Q}}=\overline{\mathbf{Q}}^{0}+\overline{\mathbf{Q}}^{(F)}+\overline{\mathbf{Q}}^{(k)}+\overline{\mathbf{Q}}^{(\Xi)}
$$

avec:

$$
\overline{\mathbf{Q}}^{0}=\int d^{3} p \frac{\mathbf{p} \otimes \mathbf{p}}{m} g(\mathbf{p})
$$

Les trois autres termes sont donnés par les équations (12) à (14).Nous allons successivement calculer chaque contribution, puis faire la somme pour obtenir $\mathbf{Q}$.

(i) Pour calculer la composante $\bar{Q}_{i j}^{0}$, par exemple, nous usons du même type de procédé qu'au $\S 2.2$ en écrivant:

$$
\begin{aligned}
\bar{Q}_{i j}^{0}=\int d^{3} p \frac{p_{i} p_{j}}{m} g(\mathbf{p}) & =\int d^{3} p \frac{1}{m}\left(p_{i}-m V_{i}\right)\left(p_{j}-m V_{j}\right) g(\mathbf{p}) \\
& +\int d^{3} p\left(V_{i} p_{j}+p_{i} V_{j}\right) g(\mathbf{p}) \\
& -\int d^{3} p m V_{i} V_{j} g(\mathbf{p})
\end{aligned}
$$

En utilisant les relations (8) et (9), on voit immédiatement qu'au second membre de l'équation (C3-3) la deuxième intégrale vaut:

$$
2 m N V_{i} V_{j}
$$

et la troisième:

$$
-m N V_{i} V_{j}
$$

Pour évaluer la première, nous utilisons l'expression $(29)$ pour $g^{e q}(\mathbf{p})$ et le changement de variable (27); elle se décompose alors en:

$$
\begin{aligned}
& \frac{1}{m} \int d^{3} p^{\prime} p_{i}^{\prime} p_{j}^{\prime} \Phi_{m}\left(\mathbf{p}^{\prime}\right) \\
+ & \frac{1}{m} \int d^{3} p^{\prime} \int d^{3} q p_{i}^{\prime} p_{j}^{\prime} \Phi_{m}\left(\mathbf{p}^{\prime}\right) \Phi_{m}\left(\mathbf{p}^{\prime}-\mathbf{q}\right)
\end{aligned}
$$

A cause de la symétrie sphérique des fonctions $\Phi_{m}$, les seuls termes non nuls sont ceux où $i$ et $j$ sont égaux, et l'expression ci-dessus se réduit à:

$$
\delta_{i j} \frac{1}{m} \int d^{3} p^{\prime} \quad \frac{p^{\prime 2}}{3} g\left(\mathbf{p}^{\prime}\right)=\frac{2}{3} W \delta_{i j}
$$


Par regroupement, nous obtenons:

$$
\bar{Q}_{i j}^{0}=\frac{2}{3} W \delta_{i j}+m N V_{i} V_{j}
$$

Le tenseur $\overline{\mathbf{Q}}^{0}$ s'écrit par conséquent, en utilisant (42):

$$
\overline{\mathbf{Q}}^{0}=\left(\frac{2}{3} U-\frac{1}{3} m N V^{2}\right) \overline{\mathbf{1}}+m N \mathbf{V} \otimes \mathbf{V}
$$

(ii) Etudions à présent $\bar{Q}_{i j}^{(F)}$, qui d'après (12) vaut:

$$
\bar{Q}_{i j}^{(F)}=-\frac{1}{2 m} \int d^{3} p^{\prime \prime} \Phi_{m / 2}\left(\mathbf{p}^{\prime \prime}\right) \int d^{3} q \frac{\Delta_{F}(q)}{q}\left(3 q_{i} q_{j}-\delta_{i j} q^{2}\right) \Phi_{2 m}(\mathbf{q})
$$

Le changement de $\mathbf{q}$ en $-\mathbf{q}$ dans la seconde intégrale indique que $\bar{Q}_{i j}^{(F)}$ est nul si $i$ est différent de $j$. Lorsque $i$ égale $j$, on peut remplacer $q_{i}^{2}$ par $\frac{1}{3} q^{2}$ ce qui conduit à:

$$
\overline{\mathbf{Q}}^{(F)}=0
$$

(iii) En ce qui concerne $\bar{Q}_{i j}^{(k)}$, nous utilisons l'identité:

$$
f^{e q}\left(\mathbf{1}^{\prime}\right) f^{e q}\left(\mathbf{2}^{\prime}\right)=f^{e q}(\mathbf{p}) f^{e q}(\mathbf{p}-\mathbf{q})
$$

pour écrire (13) sous la forme:

$$
\begin{aligned}
\bar{Q}_{i j}^{(k)}=-\frac{1}{2 m} \int d^{3} p^{\prime \prime} \int d^{3} q \int & d^{2} \hat{q}^{\prime} q^{2} \Delta_{k}(q, \theta) \times \\
& \times \hat{q}_{i}\left(\hat{q}_{j}^{\prime}-\left(\hat{q} \cdot \hat{q}^{\prime}\right) \hat{q}_{j}\right) \Phi_{2 m}\left(\mathbf{p}^{\prime \prime}\right) \Phi_{m / 2}(\mathbf{q})
\end{aligned}
$$

Nous avons effectué le changement de variables (25) pour pouvoir séparer les intégrales. Nous pouvons maintenant changer $\mathbf{q}$ en $-\mathbf{q}$ (et aussi $\mathbf{q}^{\prime}$ en $-\mathbf{q}^{\prime}$ pour conserver le produit $\hat{q} \cdot \hat{q}^{\prime}$ ); la symétrie de $\Phi_{m / 2}$ annule tous les termes non diagonaux, et donne:

$$
\begin{aligned}
\bar{Q}_{i j}^{(k)} & =-\frac{N_{L}}{2 m} \int d^{3} q \int d^{2} \hat{q}^{\prime} q^{2} \Delta_{k}(q, \theta) \delta_{i j}\left(\hat{q}_{i} \hat{q}_{j}^{\prime}-\frac{1}{3}\left(\hat{q} \cdot \hat{q}^{\prime}\right)\right) \Phi_{m / 2}(\mathbf{q}) \\
& =0
\end{aligned}
$$

(iv) Nous calculons de manière analogue la contribution de $\overline{\mathbf{Q}}^{(\Xi)}$ (équation (14)):

$$
\begin{aligned}
\bar{Q}_{i j}^{(\Xi)} & =-\frac{N_{L}}{4 m} \int d^{3} q \Xi(q) q_{i} q_{j} \Phi_{2 m}(\mathbf{q}) \\
& =\delta_{i j} \frac{N_{L}}{4 m} \int d^{3} q \Xi(q) \frac{1}{3} q^{2} \Phi_{2 m}(\mathbf{q}) \\
& =-\frac{1}{3 \beta} N_{L}^{2} \Sigma_{1} \delta_{i j}
\end{aligned}
$$

En définitive, pour la solution stationnaire uniforme considérée, le tenseur ds contraintes s'écrit:

$$
\overline{\mathbf{Q}}=\left(\frac{2}{3} U-\frac{1}{3} m N V^{2}-\frac{1}{3 \beta} N_{L}^{2} \Sigma_{1}\right) \overline{\mathbf{1}}+m N \mathbf{V} \otimes \mathbf{V}
$$




\section{C3.2 COURANT DE CHALEUR}

La densité de courant $\mathbf{J}_{U}$ s'exprime comme la somme de quatre termes:

$$
\mathbf{J}_{U}=\int d^{3} p \frac{p^{2}}{2 m^{2}} \mathbf{p} g(\mathbf{p})+\mathbf{J}_{U}^{(F)}+\mathbf{J}_{U}^{(k)}+\mathbf{J}_{U}^{(\Xi)}
$$

que nous allons calculer successivement.

(i) Le premier terme s'écrit:

$$
\begin{aligned}
\int d^{3} p \frac{p^{2}}{2 m^{2}} \mathbf{p} g(\mathbf{p})= & \int d^{3} p \frac{(\mathbf{p}-m \mathbf{V})^{2}}{2 m^{2}}(\mathbf{p}-m \mathbf{V}) g(\mathbf{p}) \\
& +\mathbf{V} \cdot \int d^{3} p \frac{(\mathbf{p}-m \mathbf{V})^{2}}{2 m} g(\mathbf{p}) \\
& -\frac{1}{2} m V^{2} \int d^{3} p \frac{\mathbf{p}}{m} g(\mathbf{p}) \\
& +\int d^{3} p \frac{(\mathbf{p} \cdot \mathbf{V})}{m} \mathbf{p} g(\mathbf{p})
\end{aligned}
$$

La première intégrale est nulle par symétrie. La contribution du second terme vaut:

$$
W \mathbf{V}
$$

celle du troisième:

$$
-\frac{1}{2} m V^{2} m N \mathbf{V}
$$

et celle du dernier:

$$
\sum_{j} V_{j} \int d^{3} p \frac{p_{j}}{m} \mathbf{p} g(\mathbf{p})=\sum_{j} \bar{Q}_{i j}^{0} V_{j}=\bar{Q}^{0} \cdot \mathbf{V}
$$

Par regroupement, nous obtenons:

$$
\int d^{3} p \frac{p^{2}}{2 m^{2}} \mathbf{p} g(\mathbf{p})=\left(W-\frac{1}{2} m N V^{2}\right) \mathbf{V}+\overline{\mathbf{Q}}^{0} \cdot \mathbf{V}
$$

(ii) Dans l'expression (16) de $\mathbf{J}_{U}^{(F)}$, on utilise l'identité suivante:

$$
(2 \mathbf{p} \cdot \mathbf{q})=2\left(\mathbf{p}-\frac{\mathbf{q}}{2}-m \mathbf{V}\right)+2 m \mathbf{V}
$$

Le premier terme du membre de droite ne contribue pas, car il introduit l'intégrale:

$$
\int d^{3} p^{\prime \prime} \mathbf{p}^{\prime \prime} \Phi_{2 m}\left(\mathbf{p}^{\prime \prime}\right)
$$

qui est nulle. Le second donne:

$$
\mathbf{J}_{U}^{(F)}=\overline{\mathbf{Q}}^{(F)} \cdot \mathbf{V}
$$

(iii) Le calcul de $\mathbf{J}_{U}^{(k)}$ se traite de manière strictement analogue. Il vient immédiatement:

$$
\mathbf{J}_{U}^{(k)}=\overline{\mathbf{Q}}^{(k)} \cdot \mathbf{V}
$$


(iv) Considérons enfin $\mathbf{J}_{U}^{(\Xi)}$ tel qu'il est donné par la formule(18). Cette fois-ci, nous utilisons l'identité:

$$
2 \mathbf{p} \cdot \mathbf{q}-q^{2}=2\left(\mathbf{p}-\frac{\mathbf{q}}{2}-m \mathbf{V}\right) \cdot \mathbf{q}+2 m \mathbf{V} \cdot \mathbf{q} \quad(C 3-26)
$$

Elle conduit à:

$$
\mathbf{J}_{U}^{(\Xi)}=\overline{\mathbf{Q}}^{(\Xi)} \cdot \mathbf{V}
$$

Par regroupement de (C3-22), (C3-24), (C3-25) et (C3-27), nous obtenons en définitive:

$$
\mathbf{J}_{U}=\left(U-m N V^{2}\right) \mathbf{V}+\overline{\mathbf{Q}} \cdot \mathbf{V}
$$

Si nous souhaitons à présent calculer $\mathbf{J}_{T}$, il convient de rappeler que:

$$
\mathbf{J}_{T}=\mathbf{J}_{U}-U \mathbf{V}-\overline{\mathbf{P}} \cdot \mathbf{V}
$$

et aussi:

$$
\begin{aligned}
\overline{\mathbf{P}} \cdot \mathbf{V} & =\overline{\mathbf{Q}} \cdot \mathbf{V}-m N(\mathbf{V} \otimes \mathbf{V}) \cdot \mathbf{V} \\
& =\overline{\mathbf{Q}} \cdot \mathbf{V}-m N V^{2} \mathbf{V}
\end{aligned}
$$

L'ensemble des trois dernières équations ci-dessus écrites implique bien:

$$
\mathbf{J}_{T}=0
$$




\section{REFERENCES}

[1] F. Laloë , J. Physique

[2] G. Tastevin, P.J. Nacher et F. Laloë, J. Physique,.....

[3] S. Chapman and T.G. Cowling, The mathematical theory of non-uniform gases, Cambridge University Press, (1970).

[4] J.O.Hirschfelder, C.F. Curtiss and R.B. Bird, Molecular theory of gases and liquids, J. Wiley and sons (1954).

[5] E.M. Lifshitz and L.P. Pitaevskii, Landau and Lifshitz course of theoretical physics, Vol. 10, Physical kinetics, Pergamon press (1981), voir le $\S 18$.

[6] J.J. Duderstadt and W.R. Martin, Transport theory, Wiley (1979).

[7] R.L. Liboff, Introduction to the theory of kinetic equations, R.E. Krieger (1979).

[8] H. Cornille, Journ. Stat. Phys. 45, 611 (1986) et références citées.

[9] K. Huang, Statistical Mechanics, Wiley (1963), § 4.

[10] E. Beth and G.E. Uhlenbeck, Physica 4, 915, (1937). 